Meta

Journal des traducteurs

Translators' Journal

\title{
À la recherche d'un titre littéraire idéalement traduit : le cas du chinois vers le français
}

\section{Honghua Poizat-Xie et Yongzhao Zhang}

Volume 62, numéro 2, août 2017

URI : https://id.erudit.org/iderudit/1041029ar

DOI : https://doi.org/10.7202/1041029ar

Aller au sommaire du numéro

Éditeur(s)

Les Presses de l’Université de Montréal

ISSN

0026-0452 (imprimé)

1492-1421 (numérique)

Découvrir la revue

Citer cet article

Poizat-Xie, H. \& Zhang, Y. (2017). À la recherche d'un titre littéraire idéalement traduit : le cas du chinois vers le français. Meta, 62(2), 368-395.

https://doi.org/10.7202/1041029ar
Résumé de l'article

Recensant 237 ouvrages littéraires chinois traduits en français publiés par les éditeurs français entre 2000 et 2014, cette étude tente d'explorer les règles et les conventions régissant la traduction de titres littéraires du chinois au français. Elle montre la complexité du processus et les multiples défis du traducteur. Nous examinons les différents procédés employés par les traducteurs, qui sont globalement au nombre de quatre : la traduction littérale, l'adaptation, l'explicitation et l'implicitation. Parfois, plusieurs procédés sont mis au service du traitement d'un titre. Nous constatons que le choix du titre ne consiste pas toujours uniquement à saisir les mots et/ou la structure de l'original. Le traducteur essaie, par toutes sortes de stratégies, d'être fidèle à l'intention de l'auteur et à l'esprit de son texte. Par ailleurs, il prend aussi en considération les caractéristiques de la langue française et les attentes du lectorat français. Il jouit d'un assez grand espace de liberté et peut raccourcir ou rallonger un titre, en condensant ou en décomposant certains mots ou structures ; il lui arrive parfois d'en réinventer un. Notre analyse montre que la majorité des titres traduits sont pertinents.
Ce document est protégé par la loi sur le droit d'auteur. L’utilisation des services d’Érudit (y compris la reproduction) est assujettie à sa politique d'utilisation que vous pouvez consulter en ligne.

https://apropos.erudit.org/fr/usagers/politique-dutilisation/ 


\title{
À la recherche d'un titre littéraire idéalement traduit: le cas du chinois vers le français
}

\author{
HONGHUA POIZAT-XIE \\ Département des études est-asiatiques, Université de Genève, Genève, Suisse \\ grace.poizat@unige.ch \\ YONGZHAO ZHANG \\ Éditions en langues étrangères, China International Publishing Group, Beijing, China \\ zhang20032005@sina.com
}

\begin{abstract}
RÉSUMÉ
Recensant 237 ouvrages littéraires chinois traduits en français publiés par les éditeurs français entre 2000 et 2014, cette étude tente d'explorer les règles et les conventions régissant la traduction de titres littéraires du chinois au français. Elle montre la complexité du processus et les multiples défis du traducteur. Nous examinons les différents procédés employés par les traducteurs, qui sont globalement au nombre de quatre: la traduction littérale, l'adaptation, l'explicitation et l'implicitation. Parfois, plusieurs procédés sont mis au service du traitement d'un titre. Nous constatons que le choix du titre ne consiste pas toujours uniquement à saisir les mots et/ou la structure de l'original. Le traducteur essaie, par toutes sortes de stratégies, d'être fidèle à l'intention de l'auteur et à l'esprit de son texte. Par ailleurs, il prend aussi en considération les caractéristiques de la langue française et les attentes du lectorat français. Il jouit d'un assez grand espace de liberté et peut raccourcir ou rallonger un titre, en condensant ou en décomposant certains mots ou structures; il lui arrive parfois d'en réinventer un. Notre analyse montre que la majorité des titres traduits sont pertinents.
\end{abstract}

\begin{abstract}
The following study inventories the titles of a total of 237 Chinese literary works in French translation published in France between 2000 and 2014, and attempts to explore the rules and conventions governing the translation of literary titles from Chinese to French. It demonstrates the complexity of this process and the multiple challenges devolving on the translator. We examine the four methods of translating a title: literal translation, adaptation, explicitation, and implicitation. Sometimes several of these methods may be employed in the translating of a single title. We will see that the choice of a title is not always to convert into French the words and/or the structure of the title in Chinese. Through the use of various strategies, the translator strives to be faithful to both the spirit of the author and his text. But the translator must also take into account the characteristics of his target language and the customs of a French readership. Here he benefits from a considerable liberty: he can shorten a title or expand it, condense or decompose certain words or structures. Sometimes he has even invented an entirely new title. Our analysis has shown that the great majority of translated titles remain apt to their subject.
\end{abstract}

\section{RESUMEN}

El presente estudio es un catálogo de los títulos de un total de 237 obras literarias chinas en traducción francesa, publicadas en Francia entre 2000 y 2014. Apunta a explorar los procedimientos que rigen la traducción de títulos literarios del chino al francés. It demonstrates the complexity of this process and the multiple challenges devolving on the translator. Examinamos los cuatro procedimientos de traducción siguientes: la traducción literal, la adaptación, el explicitación y la implicitación, pudiendo emplearse más 
de un procedimeiento en un solo título. Se verá que la elección de un título no consiste siempre en verter en francés las palabras o estructuras del título original en chino. Al utilizar varios procedimientos el traductor intenta ser fiel al propósito del autor y espíritu del texto. Por otra parte, toma en cuenta las características de la lengua francesa y las expectativas del lectorado francés. Goza de un gran margen de libertad: puede acortar o alargar un título condensando o descomponiendo palabras o estructuras. A veces crea una palabra. Nuestro estudio muestra la pertinencia de la mayoría de los títulos traducidos.

\section{MOTS-CLÉS/ KEYWORDS/PALABRAS CLAVE}

titre littéraire, traduction, procédé, chinois, français literary title, translation, method, chinese, french

título literario, traducción, procedimiento, chino, francés

\section{Introduction}

Comme les yeux pour une personne, le titre est la fenêtre d'une œuvre permettant d'ouvrir le monde de celle-ci. Dans bien des cas, le titre ne renvoie pas seulement à une œuvre, mais aussi à l'écrivain lui-même. Aucun lecteur ne peut évoquer Guerre et Paix sans penser à Tolstoï, Le Rouge et le Noir, à Stendhal, ou Le vieil homme et la mer, à Hemingway. Et vice-versa. Un bon titre synthétise le contenu de l'œuvre et renvoie à l'intention créatrice de l'écrivain. Il accompagne le lecteur d'un bout à l'autre de l'œuvre et joue un rôle fondamental dans son activité mentale lorsqu'il tente d'appréhender la pensée de l'auteur et la signification de l'œuvre. Umberto Eco a si bien dit: «Un titre est déjà la clé d’un commentaire» (Eco 1979/1985: 7).

Le titre peut être également un signal qui accroche le lecteur potentiel, car sur la couverture du livre il réalise le premier contact de l'œuvre avec le lecteur. Un titre attrayant peut inciter des personnes en balade dans une librairie à lire le résumé du livre puis, éventuellement, à l'acheter. Il peut donner envie de lire au lecteur et restera gravé dans son esprit une fois la lecture terminée. Les études montrent que le titre exerce une grande influence sur la lecture ${ }^{1}$.

La traduction d'un titre représente donc un enjeu important pour le traducteur. Elle doit «non seulement respecter les normes, mais aussi tenir compte de l'intertextualité des titres du système de réception» (Malingret 1998: 398). L'étude de l'art de la traduction des titres joue un rôle d'une grande importance dans la recherche sur la traduction littéraire en général. En faisant un état des lieux de la traduction en français de 237 titres d'œuvres littéraires chinoises publiées par les éditeurs français entre 2000 et $2014^{2}$, la présente recherche tente de dégager les règles et les conventions régissant depuis ces quinze dernières années la traduction des titres. Nous espérons que notre travail puisse apporter quelques éclaircissements et servir de guide aux traducteurs qui s'engagent dans leur mission.

En comparant les 237 titres originaux et leurs versions françaises, nous avons pu distinguer les quatre procédés les plus utilisés: 1) la traduction littérale, 2) l'adaptation, 3) l'explicitation, et 4) l'implicitation. Le tableau I permet de comparer la fréquence de chaque procédé par rapport à l'ensemble.

Dans nos statistiques, pour simplifier le comptage, nous comptons un procédé par titre. Lorsque plusieurs procédés sont appliqués, nous choisissons celui qui nous paraît le plus évident ou le plus important. Par exemple, dans La nuit quand tu me manques, j'peux rien faire: panorama du village des Wen de Cai Naiqian, la première 
partie traduit littéralement le titre d'origine (Dao heiye xiang ni mei banfa, arriver- nuit noir-penser-tu-ne pas avoir-solution), mais nous le plaçons dans la catégorie de l'explicitation. La même chose pour L'empire de l'absurde, ou Dix ans de la vie de gens ordinaires de Feng Jicai. Malgré une partie fidèle à l'origine (Yibaige ren de shi nian, 100-homme-DE-10-an), nous le rangeons dans la catégorie de l'adaptation-substitution.

Dans notre analyse, lorsque nous jugeons un titre traduit, nous sommes tout à fait conscients que notre jugement peut être subjectif. Les critères destinés à évaluer la qualité des titres traduits varient d'un chercheur à l'autre. Shen (2013: 199-200) par exemple, montre bien la difficulté d'expertiser une bonne traduction de titre à l'aide de différentes versions chinoises de Gone with the Wind de Margaret Mitchell. Certains chercheurs établissent des «degrés» mathématiques pour cette évaluation (Zhang 2001). Billeter (2014: 115-116) considère la «beauté » comme critère pour juger une traduction réussie, même s'il reconnaît que l'on peut analyser seulement jusqu'à un certain point. Selon l'auteur, la «beauté» est "la qualité de ce qui est devenu immédiatement intelligible»; elle "produit un effet», elle est «un effet», elle est «simple dans son effet». Cette notion de beauté est, à nos yeux, trop arbitraire et abstraite, donc peu probante. Quant à nous, nous nous attacherons essentiellement à deux aspects: premièrement, si le titre traduit est fidèle à l'esprit de l'auteur ou à l'essence du texte; deuxièmement, s'il se montre efficace, éclairant et poétique. Si nous considérons le titre en chinois du chef-d'œuvre de Marcel Proust, $\grave{A}$ la recherche du temps perdu, comme Zhuiyi sishui nianhua (追忆似水年华, mémoriser-commeeau-année), nous ne pouvons que nous émerveiller face à la splendeur et quasi perfection de cette traduction.

TABleau I

Répartition des procédés de traduction de titres d'œuvres littéraires

\begin{tabular}{|l|c|c|}
\hline \multicolumn{2}{|l|}{ Nombre de titres } & 237 \\
\hline \multirow{3}{*}{ Procédés } & Traduction littérale & $124(53 \%)$ \\
\cline { 2 - 3 } & Adaptation & $74(31 \%)$ \\
\cline { 2 - 3 } & Explicitation & $24(10 \%)$ \\
\cline { 2 - 3 } & Implicitation & $15(6 \%)$ \\
\hline
\end{tabular}

\section{Traduction littérale}

Par traduction littérale, nous entendons une traduction lexicalement et syntaxiquement fidèle à l'original. Comme tout auteur accorde une grande attention au titre de son œuvre, nombre de traducteurs se contentent de le traduire littéralement. Parmi les 237 titres, 124 sont dans cette catégorie, soit $53 \%$. Nous pouvons constater que les traducteurs ne changent pas à la légère les titres originaux. Cela confirme aussi le résultat d'une étude de Wang (1994: 24-25), qui montre que $70 \%$ des traductions de titres littéraires en langues occidentales vers le chinois, durant la période allant de 1949 aux années quatre-vingt, recourent à la traduction littérale. Certaines œuvres, retraduites durant cette période, ont reçu un titre plus proche de l'original.

La conversion des codes linguistiques entre deux langues comme le chinois et le français s'avère souvent complexe et nébuleuse, du fait qu'il existe d'énormes différences sur les plans morphologique et syntaxique. Ainsi, Yu (2008: 69-70) a-t-il obtenu 32 traductions en chinois de The Call of the Wild par Jack London, avec cinq 
titres différents qui pourtant relèvent de la traduction littérale. Ceci est dû au fait que les mots en langue chinoise correspondant à call et wild sont nombreux ${ }^{3}$. Dans le cadre de notre étude, les problématiques les plus manifestes concernent le choix de la nature de la structure (nominale ou verbale), l'article (présent ou absent, défini ou indéfini), et le nombre (singulier ou pluriel).

Parmi les 124 titres traduits littéralement, nous pouvons distinguer deux structures utilisées: nominale pour l'écrasante majorité (111) et verbale pour les 13 titres restants (y compris la structure phrastique), soit respectivement $90 \%$ et $10 \%$ (voir le tableau II). Si nous examinons l'ensemble des 237 titres traduits, 214 sont de nature nominale, représentant aussi $90 \%$; seuls 19 sont verbaux et phrastiques (8\%) et 7 sont prépositionnels. Or, parmi les titres originaux, 187 sont nominaux et 50 verbaux, soit respectivement $79 \%$ et $21 \%$. Pour les titres verbaux avant et après la traduction, nous constatons donc un écart de $13 \%$. La majorité des traducteurs optent donc pour une structure nominale pour rendre les titres chinois en français.

Dans les titres nominaux, nous examinons l'emploi de l'article lié au nom, notamment les raisons qui poussent le traducteur à choisir un nom sans article. Quant aux titres verbaux, ils peuvent être sous la forme d'un verbe à l'infinitif ou d'une phrase.

TABLEAU II

Répartition des titres traduits littéralement selon leur nature grammaticale

\begin{tabular}{|c|c|c|c|c|}
\hline \multicolumn{2}{|c|}{$\begin{array}{l}\text { Nombre et pourcentage par } \\
\text { rapport aux } 237 \text { titres }\end{array}$} & $124(53 \%)$ & & \\
\hline \multirow{7}{*}{ Nature du titre } & \multirow{5}{*}{$\begin{array}{l}\text { Structure } \\
\text { nominale }\end{array}$} & \multirow{5}{*}{$111(90 \%)$} & Article défini & $49(44 \%)$ \\
\hline & & & Sans article & $43(39 \%)$ \\
\hline & & & Article indéfini & $6(5 \%)$ \\
\hline & & & Numérale & $5(5 \%)$ \\
\hline & & & Nom propre & $8(7 \%)$ \\
\hline & \multirow{2}{*}{$\begin{array}{l}\text { Structure } \\
\text { verbale }\end{array}$} & \multirow{2}{*}{$13(10 \%)$} & Verbe à l'infinitif & $6(46 \%)$ \\
\hline & & & Structure phrastique & $7(54 \%)$ \\
\hline
\end{tabular}

\subsection{Structure nominale}

Le chinois relève de la famille des langues sino-tibétaines qui manquent de marqueurs morphologiques, tandis que le français appartient à celle des langues indoeuropéennes qui recourent largement aux modifications morphologiques. Bien que la structure nominale existe bel et bien dans les deux langues, le chinois ne connaît guère le marqueur du pluriel; l'article n'y existe pas. Lorsqu'on traduit un nom chinois vers le français, il faut interroger à la fois deux aspects: son nombre (singulier/pluriel) et son ajout ou non d'un article. Si l'on décide de mettre un article, il faut encore choisir quel type d'article à utiliser: défini, indéfini, ou bien partitif. Ce problème d'articles se pose également dans la structure «numéral + nom».

Selon la fréquence, nous distinguons deux groupes largement dominants: le groupe des noms précédés par un article défini et le groupe des noms sans article.

Les trois groupes restants, dont chaque groupe compte moins de dix titres, sont nettement minoritaires par rapport à l'ensemble de titres nominaux (111): 6 noms précédés d'un article indéfini, 5 noms précédés d'un nombre et 8 noms propres. 


\subsubsection{Article défini + nom}

Nous comptons 49 structures sous forme «article défini + nom» en français, soit $44 \%$, c'est-à-dire presque la moitié. Si nous examinons l'ensemble, 136 titres sur 237 contiennent une structure nominale dont 73 avec un article défini, soit $54 \%$, ce qui n'est pas très loin de $44 \%$. C'est la formule la plus courante dans les structures nominales. Par exemple, Le monde futur (Weilai shijie, futur-monde) ${ }^{4}$ de Wang Xiaobo; Le veau (Niu) et La belle à dos d'âne dans l'avenue de Chang'an (Chang'an dadao shang de qi lü meiren, Chang'an-avenue-sur-DE-enjamber-âne-beauté) de Mo Yan, La berge (He an, rivière-bord) de Su Tong et La philosophie de Lao Zhang (Lao Zhang de Zhexue, Lao Zhang-DE-philosophie) de Lao She. Dans ces titres, tous les noms sont précédés d'un article défini parce qu'ils ont généralement une portée spécifique dans le contexte du roman. Larticle défini remplit ici sa fonction de spécification et de singularisation. Le monde n'est pas n'importe quel monde, mais un monde défini, suivant une vision unique que le lecteur va découvrir. Le veau n'est pas un veau quelconque, mais un veau déterminé et également le héros du roman. De même, la berge est la berge singulière que l'auteur va nous décrire. Quant à la philosophie, elle est de Lao Zhang, donc spécifique.

Il est intéressant de remarquer que l'oeuvre Nian yue ri (année-mois-jour) de Yan Lianke est traduit par Les jours, les mois, les années au lieu de «Années, mois, jours» tel qu'écrit dans le titre original. Cette modification s'explique par l'ordre logique différent dans les deux langues concernées: l'ordre en chinois va du plus grand au plus petit, alors que celui du français privilégie le sens inverse, du plus petit au plus grand. Les articles définis au pluriel répétés jouent ici un rôle d'accentuation et permettent de mettre en relief une hiérarchie temporelle.

Deux autres titres ont opté pour la modification de l'ordre syntaxique, consistant à inverser la position du modifié et du modifiant. Il nous semble qu'Une folie d'orchidées est plus poétique et mystérieux que la traduction littérale «Orchidées en folie» (Fengkuang de junzilan, folie-DE-orchidée, de Zhang Xinxin). De plus, le roman ne traite pas vraiment d'orchidées, ces dernières restent symboliques. Par contre, la signification littérale du titre d'origine «Les bas-fonds de l'empire» (Diguo diceng, empire-base, de Liao Yiwu) n'équivaut pas à celle de L'empire des bas-fonds. Nous ne voyons pas la raison particulière de ce changement de la part du traducteur.

\subsubsection{Nom (sans article)}

Sur 111 structures, 43 restent sans article, soit 39\%. La proportion des titres sans article dans l'ensemble permet aussi de confirmer ce pourcentage: 57 des 136 structures pouvant avoir un article restent sans article, ce qui représente aussi $42 \%$. C'est également une structure pour laquelle les traducteurs optent souvent.

Il nous semble que, si un nom est employé sans article, c'est essentiellement parce qu'il n'est pas spécifique, qu'il représente un cas comme les autres ou qu'il revêt un certain sens symbolique. D’après Grévisse (1993: 882), l'absence de l'article est observée "souvent dans les phrases adverbales servant d'inscription, de titre, d'adresse, etc.», mais l'auteur ne précise pas les conditions pour une absence de l'article. Un peu plus loin, il estime même que «toute l'évolution va dans un même sens: rendre la présence de l'article de plus en plus automatique» (1993: 883). Les noms employés dans les titres sont autorisés à rester sans article, mais si nous examinons de près la tradition de la littérature française, nous pourrons nous apercevoir que ce cas est très peu courant. 
Nous avons effectué un petit comptage sur une de nos bibliothèques universitaires contenant principalement des ouvrages littéraires en français: sur un total de 109 , seuls 5 titres sont composés de noms au pluriel sans article, et 12 de noms au singulier sans article. Les titres sans article ne représentent donc que $16 \%$ de la totalité, pourcentage fort inférieur aux $39 \%$ de nos titres traduits ( $23 \%$ de moins). De plus, parmi ces titres français sans article, une grande partie se rapporte à l'Asie, comme Stupeur et tremblement d'Amélie Nothomb, Passagère du silence de Fabienne Verdier, Chronique japonaise de Nicolas Bouvier, Opium et Neige de Maxence Fermine. Cela confirme, dans une certaine mesure, notre intuition: les titres sans article sont exotiques, en particulier dotés d'un exotisme oriental. Il se peut aussi que ce soit une influence des titres littéraires en anglais qui emploient peu d'articles.

Lorsque le premier nom est au pluriel et indéfini, il est généralement privé d'article. Par exemple, Cris dans la bruine (Zai xiyu zhong huhuan, dans-bruinemilieu-crier) de Yu Hua, Dés de poulet façon mégère (Pofu jiding, mégère-dés de poulet) de Liu Xinwu et Reflets sur la rivière obscure (Hei'an heliu shang de shanguang, obscur-rivière-sur-DE-reflet) de Qiu Huadong. Un seul nom au pluriel précédé d'un article indéfini, c'est Des yeux gris clair (Dan huise de yanzhu, clair-gris-DE-prunelle) de Wang Meng. La raison de cet ajout n'est cependant pas sémantique, mais plutôt phonétique: comme le mot yeux commence par une semi-voyelle, on le précède de des pour faciliter la prononciation. La comparaison entre Personnages (Chuanqi, légende) de Feng Jicai et Les mandarins (Guanren) de Liu Zhenyun est particulièrement parlante. Le premier est un recueil de nouvelles dont chacune décrit un personnage. Ces personnages sont représentatifs, mais non exhaustifs, et sans parti pris, de la société traditionnelle chinoise. De plus, l'absence d'article diffuse un parfum de hasard et de mystère. Dans le deuxième cas, le traducteur choisit de mettre un article défini, car l'intention de l'auteur porte sur la mise en évidence d'un ensemble de caractéristiques des mandarins.

Lorsque le nom est au singulier et sans article, les effets qu'il provoque peuvent être particuliers. Voyons deux exemples. D'abord, Cataclysme (Zai bian, catastrophechanger) de Liu Qingbang. Comparé à «Le cataclysme», le mot sans article semble plus tragique et donne plus de marge d'imagination aux lecteurs. Le cataclysme risque facilement d'être mal compris et d'être considéré comme un livre traitant le cataclysme ou décrivant un cataclysme défini. Quant à "cataclysme» sans article, il pourrait faire sentir aux lecteurs qu'il s'agit d'un cataclysme concret et spécifique. Ensuite, Poisson à face humaine (Ren mian yu, humain-visage-poisson) de Liu Xinwu. Ici, le poisson à face humaine, n'importe lequel, doté d'un sens symbolique, représente les souvenirs de la protagoniste qui font surface comme dans l'étang où elle se rendait durant son enfance. L'un comme l'autre, Cataclysme et Poisson à face humaine ne sont pas des termes originellement teintés d'une couleur littéraire, mais ils sont dotés d'une finesse et d'un d'espace imaginaire grâce à leur emploi sans article, si bien que ces deux termes, complètement neutres à l'origine, s'animent soudainement.

\subsubsection{Article indéfini + nom, Nombre + nom, Nom propre}

Les autres formes de titres nominaux sont peu représentatives, car nous comptons seulement 6 structures en "article indéfini + nom», 5 en «nombre + nom» et 8 en nom propre. Dans le premier cas, l'article indéfini est toujours un/une. Il peut avoir deux fonctions: soit article indéfini, soit nombre. Citons parmi d'autres Une fille de 
la faim (Ji'e de nü'er, faim-DE-fille) de Hong Ying et Un vol (Feixing, voler) de Yu Jian. Si le traducteur emploie un article indéfini, cela signifie logiquement que le nom est indéfini. Une fille de la faim est un roman autobiographique qui raconte l'histoire d'une jeune fille et sa famille, mais il appartient également à une époque, à une région, et en définitive à une nation ${ }^{5}$. Par conséquent, il s'agit d'une histoire concernant une fille particulière, mais aussi celle d'une fille quelconque de cette terre. Un vol, long poème narrant le déroulement d'un vol, évoque un voyage à la fois réel et symbolique. Un vol, comme n'importe quel vol, traverse l'espace et le temps; il est en même temps un vol particulier, permettant au poète de dialoguer avec l'Himalaya et T.S. Eliot, et de revisiter la Révolution culturelle et le maoïsme.

Dans le deuxième cas, les 5 structures numérales en «nombre + nom» peuvent, selon la présence ou non de l'article, se subdiviser en deux catégories: structures avec article défini et structures sans article. Pour les premières, citons Les quatre livres (Si shu, quatre-livre) de Yan Lianke. Il s'agit de quatre livres spécifiques, d'où l'emploi d'un article. En fait, l'auteur a emprunté l'appellation "Zhongguo si da jingdian», soit «Les quatre classiques». Le livre laisse ici entendre une oeuvre classique. Par ailleurs, Yige ren de shengjing (un-homme-DE-Bible) de Gao Xingjian est rendu par Le livre d'un homme seul. Shengjing, signifiant la Bible, est devenu le livre, le classique. D'autres exemples, Les trois portes (San chong men) de Han Han, et La douzième nuit (Di shi'er ye) de Tie Ning, dans lesquels les articles jouent un rôle déterminant, et dont les histoires se déroulent respectivement autour des «trois portes» et de «la douzième nuit ».

Deux cas n'ont pas d'article: Quatre générations sous le même toit (Si shi tong tang, quatre-génération-même-maison) de Lao She et Quarante et un coups de canon (Sishiyi pao, 41-canon) de Mo Yan. Le grand roman de Lao She a comme titre une expression idiomatique chinoise. Il décrit la vie des familles pékinoises vivant dans l'étroit hutong sous l'occupation japonaise. Quatre générations sous le même toit était le mode de vie traditionnel. Il s'agit d'une désignation générale pour les quatre générations. Le deuxième cas, Quarante et un coups de canon, a un lien étroit avec la structure du roman: l'intrigue se déroule au rythme de chaque coup de canon. Au début de l'histoire, les lecteurs ne savent pas combien de coups se produiront, le chiffre sans article donne donc un effet progressif. Si l'on y ajoute l'article défini les, le titre risquerait de ne plus pouvoir surprendre et d'être moins compact.

Quant au troisième cas, les 8 titres sont composés de noms propres qui peuvent aussi être regroupés en deux catégories, selon l'origine du nom propre: soit comme Pentium III (Penteng III) de Lao Niu ou encore Madame Liu (Liushi nü) et Madame Yang (Yangshi nü) de Zhang Yihe, soit un nom commun à l'usage de nom propre, tel que Bronze et Tournesol (Qingtong Kuihua) de Cao Wenxuan. La première catégorie ne pose pas de problème au lecteur, tandis que la deuxième reflète la particularité des prénoms chinois qui sont presque toujours chargés de connotations. Le traitement d'un nom propre se révèle souvent un défi pour le traducteur. Nous étudions dans un autre article le phénomène des noms propres dans les titres.

\subsubsection{Nombre: singulier ou pluriel}

Entre deux langues aussi éloignées que le chinois et le français, même les aspects les plus simples et clairs peuvent devenir compliqués. La question du nombre en est un exemple. Puisque le chinois ne possède pas de marqueur de nombre, nous ne pouvons 
pas savoir si les noms du titre sont au singulier ou au pluriel. Le traducteur décide selon deux principes: soit selon le contenu de l'œuvre, soit selon les règles usuelles de la langue française. Pour le premier cas, nous pouvons citer Le petit lettré de Tianjin et Personnages de Feng Jicai. Par les titres originaux en chinois, Sushi qiren et Chuanqi, nous ne pouvons savoir le singulier ou le pluriel dans le titre. C'est à travers la lecture des œuvres que nous apprenons qu'il s'agit d'un qi ren (surprenanthomme) et de plusieurs chuanqi. Parfois, dans le même titre, on a les deux formes, tel que le recueil de poèmes Rêve blanc, âmes noires (Bai meng, Hei hun, blanc-rêvenoir-âme) de Ma Desheng. Le choix du nombre ici dépend entièrement du contenu de l'œuvre.

Pour le deuxième cas, ce sont les exigences de la langue française qui dictent la loi. Le traducteur ne choisit en réalité pas vraiment. Si le mot Jumeaux s'emploie pour traduire Liansheng (de Diao Dou), il est obligatoirement sous forme plurielle. La même raison pour Poussière et sueur de Liu Xinwu ou Poussière rouge de Mo Ren où les mots poussière et sueur sont généralement sous forme singulière.

Comme la grande majorité des noms possèdent les deux formes, le traducteur doit procéder au choix selon le texte et le contexte.

\subsection{Structure verbale}

Peu de titres en français sont de nature verbale (seulement $10 \%)$. Avec un écart de $14 \%$ avant et après traduction, transformer une structure verbale en structure nominale constitue l'une des pratiques que le traducteur a régulièrement envisagées.

La structure verbale comprend le verbe à l'infinitif (au nombre de 6) et la séquence phrastique (au nombre de 7). Le roman Sha fu (tuer-mari) de Li Ang, transposé mot à mot en Tuer son mari, semble logique. Servir le peuple pour rendre Wei renmin fuwu (pour-peuple-servir) de Yan Lianke est parfait, car presque tout le monde connait ce slogan du communisme chinois. En revanche deux titres, Accrocher les coins de la bouche au bord des oreilles (Ba zui jiao gua zai er bian, BA-bouchecoin-accrocher-oreille-bord) et Sauver une vie (Jiu ming, sauver-vie), tous deux de Dong $\mathrm{Xi}$, nous rendent un peu perplexes. Si le second est à peu près admissible, le premier en tant que titre nous semble assez bizarre, à cause de sa longueur et de sa nature verbale. Le traducteur souhaite certes rester fidèle à l'original, mais un tel titre en français s'avère difficilement acceptable. Il vaudrait certainement mieux chercher une autre solution. Quant à Se souvenir de 1942 (Wengu 1942) de Liu Zhenyun, cette traduction ne nous semble pas la meilleure solution. La raison est certainement due à sa nature verbale. L'écrivain évoque ici une grande famine survenue en 1942, il questionne les survivants et essaie de faire revivre leurs souvenirs. Pourquoi pas Souvenirs de 1942 ou, encore mieux, Retour en 1942? D'ailleurs, son titre en anglais, Back to 1942, est meilleur que celui en français.

Les séquences phrastiques, comparées aux verbes à l'infinitif, nous semblent bien plus libres, si bien que la totalité des titres originellement phrastiques est rendue par une structure similaire en français. Ainsi, nous trouvons $\mathrm{Tu}$ es une rivière pour $\mathrm{Ni}$ shi yitiao he (tu-être-une-rivière) de Chi Li, Le maître a de plus en plus d'humour pour Shifu yuelaiyue youmo (maître-de plus en plus-humour) de Mo Yan, Et tout ce qui reste est pour toi pour Shengxia de dou shuyu ni (rester-DE-tout-appartenir-toi) de Xu Xing. 
En résumé, la traduction littérale est le premier choix des traducteurs. Les titres nominaux priment largement sur les verbaux, et la majorité des titres nominaux soit emploie un article défini, soit reste sans article. Dans ce qui suit, nous allons montrer dans quels cas le traducteur abandonne la traduction littéraire et opte pour une tradaptation, une réduction ou un ajout.

\section{Adaptation}

Par adaptation, nous entendons un procédé «qui donne préséance aux thèmes traités dans le texte de départ, indépendamment de sa forme» (Delisle et al. 1999: 8). Le traducteur peut proposer un titre complètement nouveau, ce que nous appelons substitution. Il peut aussi apporter une modification lexicale et/ou effectuer une recatégorisation; ou encore moduler l'original en gardant sa signification essentielle. Sur les 237 titres traduits, 74 sont dans ce cas, soit $31 \%$. Le tableau III montre la répartition des quatre méthodes.

TABleau III

Répartition des titres par adaptation

\begin{tabular}{|l|l|c|}
\hline Nombre et pourcentage par rapport aux 237 titres & $74(31 \%)$ \\
\hline \multirow{4}{*}{ Méthodes } & Substitution & $32(43 \%)$ \\
\cline { 2 - 3 } & Modification lexicale & $17(23 \%)$ \\
\cline { 2 - 3 } & Recatégorisation & $21(28 \%)$ \\
\cline { 2 - 3 } & Reformulation & $4(6 \%)$ \\
\hline
\end{tabular}

\subsection{Substitution}

La substitution consiste à abandonner le titre original au profit d'un nouveau titre. Strictement parlant, cela ne relève plus du cadre de la traduction, mais de celui d'une recréation du traducteur. Le recours à cette méthode nécessite un certain courage, car la compréhension du traducteur s'avère, en fin de compte, personnelle et subjective. Elle ne se conforme pas forcément à l'intention de l'auteur. Nous sommes de l'avis de la majorité des chercheurs, que la traduction est également un processus de recréation artistique et que chaque traduction ne représente qu'une interprétation individuelle. Bien évidemment, cette recréation exige des conditions préalables et des restrictions, à savoir qu'elle doit se faire sous le principe d'une considération approfondie de l'œuvre originale. Lorsque le traducteur donne avec audace un nouveau titre à l'œuvre, il doit redoubler de prudence, tout en assurant la valeur et l'effet du titre qu'il réinvente. Il ne doit pas ne citer que des mots isolés sans tenir compte du contexte, ni prendre une partie pour l'ensemble, encore moins se couper du texte original. Il ne doit pas non plus donner libre cours excessivement à sa créativité, ni débiter de belles phrases pour plaire au public, ni faire une traduction kitsch ${ }^{6}$.

Parmi les 74 titres adaptés, 32 ont été remplacés par un nouveau titre, c'est-à-dire $43 \%$, soit un pourcentage très élevé7. Cela signifie que sur l'ensemble des 237 titres, $14 \%$ ont changé complètement de visage.

Voyons quelques exemples à travers lesquels nous tentons de comprendre dans quelles circonstances les traducteurs décident d'effectuer un changement radical de titre. Tuina, le roman de Bi Feiyu, relate les ténèbres et la lumière au fond du cœur 
d'un groupe de masseurs non-voyants. Le terme tuina, utilisé dans la médecine traditionnelle chinoise, peut être traduit par «Massage chinois» ou "Massage de la médecine chinoise», mais cela risque d'être mal compris par un lecteur occidental. Les protagonistes du roman étant tous aveugles, le traducteur a choisi un nouveau titre, Les aveugles. Ce titre, très proche du sujet de l'œuvre, paraît meilleur qu'une traduction littérale. Nanfang (Le Sud) de Wang Chao raconte l'histoire d'un homme du Sud ayant quitté sa ville natale pour venir gagner sa vie à Beijing. Il y fait connaissance d'une jeune veuve. Celle-ci, originaire de la banlieue de Beijing, est étalagiste. Le coup de foudre met ces deux personnages ensemble, ils s'entraident et se démènent pour faire du commerce. Si l'on traduisait littéralement ce titre par «Le Sud», ce serait assez médiocre. Alors que le titre français Homme du Sud, femme du Nord, à l'aide d'une rhétorique d'opposition et d'un parallélisme rythmique, réussit à donner lieu à un contraste frappant, tout en indiquant l'origine des deux protagonistes de l'histoire. Duimian you ren (en face-avoir-homme) de Dai Lai, littéralement «Il y a quelqu'un en face», décrit la vie amoureuse d'un jeune citadin ordinaire. Le titre en chinois se rapporte au fait que le protagoniste a toujours le sentiment que quelqu'un filme en cachette les séquences de son quotidien. Le titre français, L'insecte sur la toile, porte l'attention plutôt sur les images de la vie passive devant l'écran de l'ordinateur secret du protagoniste. Pareil à une mouche retenue par une toile d'araignée, il n'est qu'un insecte dérisoire retenu par Internet. Le nouveau titre fait pressentir plus ou moins le suspense d'un roman policier, éveillant d'emblée la curiosité des lecteurs.

Quelques titres nouveaux méritent par ailleurs une discussion. Prenons Wuya (corbeau) de Jiu Dan. Le roman décrit la vie atypique d'un groupe de jeunes étudiantes à Singapour. Venues de Chine continentale, elles vivent de la prostitution dans l'espoir de se procurer une carte de résident permanent. Les corbeaux immigrés à Singapour, le sens de Wuya, symbolisent ces jeunes filles. Elles ne plaisent pas aux autochtones, mais se battent obstinément pour leur existence et leur accroissement. Les Singapouriens appellent ces femmes Long nü (dragon-fille), d'où le titre français Filles-dragons. À nos yeux, le nouveau titre est discutable, car il provient du point de vue des Singapouriens, et non de celui de l'auteur, ni de celui de l'héroïne du roman (à savoir que l'auteur narre l'histoire à la première personne), encore moins de celui des lecteurs occidentaux. Il se peut que la traduction littérale «Corbeaux» soit plus proche de l'intention de l'auteur. Étant un oiseau de mauvais augure, détesté par les Chinois, le corbeau est plus qualifié pour représenter ces femmes qui luttent pour leur vie au moyen de la prostitution.

Il en ressort que l'option d'un nouveau titre, tout comme la réduction ou l'explicitation, a pour but de faciliter la compréhension, en évitant d'éventuelles difficultés ou malentendus causés par les deux langues ou les deux cultures. Nous partageons l'idée de Yang (1996: 43) qui affirme, dans son étude sur les titres traduits en anglais de cent histoires drôles de la Chine antique, que la traduction des titres doit être «vivante et expressive», "précise et concise», notamment "éviter les obstacles culturels dans le transfert entre deux langues, rajuster le mode d'expression du texte traduit en fonction de l'habitude mentale et culturelle des lecteurs de la langue d'arrivée, afin que les titres soient clairs et intelligibles ${ }^{8} »$. 


\subsection{Modification lexicale}

Certains traducteurs effectuent des modifications lexicales que l'on peut qualifier de légères en traduisant le titre. Certains modifient un mot dans l'original. Dans Les lumières de Hongkong (Xianggang de qing yu ai, Hongkong-DE-sentiment-et-amour) de Wang Anyi, l'emploi de lumières à la place de Sentiments et amour s'avère plus troublant et plus poétique. Le mot lumières symbolise mieux l'amour provisoire et à caractère commercial des protagonistes. Si nous comparons Voyage au pays de l'oubli et Yiwang zhe zhi lu (oublier-homme-de-chemin), littéralement "Le chemin de ceux qui oublient», de Qiu Huadong, nous découvrons la complexité et l'intelligence dans ce jeu de modification lexicale. Le roman raconte le voyage de mémoire involontaire d'un homme qui a oublié son passé. Le retour progressif de la mémoire lui fait prendre conscience de l'amour éphémère. À la fin du voyage, l'oubli lui revient comme l'unique délivrance possible. Puisque l'on ne trouve pas un mot convenable pour yiwangzhe (les personnes qui oublient), au pays de l'oubli semble une bonne alternative. Et voyage au lieu de chemin paraît plus symbolique. Quant à Songeant à mon père (Wo yu fu bei, moi-et-père-génération) de Yan Lianke, son titre original en chinois était «Mon père et moi». C'est le traducteur qui l'a modifié. Il s'agit en effet d'un roman-fleuve rempli de souvenirs de l'auteur: son pays natal est inquiétant et inoubliable, son père souffrant et inflexible. Le troisième chapitre de l'œuvre est justement intitulé Xiangnian fuqin, Songeant à mon père.

\subsection{Recatégorisation}

La recatégorisation est un procédé qui «consiste à établir une équivalence par un changement de catégorie grammaticale» (Delisle et al. 1999: 65). Cette opération a concerné 21 titres. La recatégorisation s'applique le plus souvent à changer un verbe en nom, puisque les titres français sont rarement verbaux, comme nous l'avons montré dans la section 1.2. On peut citer que Jiejue (résoudre) de Diao Dou est rendu par Solutions, Shu qing (exprimer-sentiment) de Feng Jicai par Sentiments, Yumou sha ren (préméditer-tuer-homme) de Chi Li par Préméditation, ou encore Tiantang you ai (paradis-avoir-amour) de Wang Chao par Au paradis, l'amour.

La recatégorisation s'applique aussi à transformer un titre nominal ou verbal en structure prépositionnelle. Ainsi, la préposition à a été ajoutée dans le titre de l'œuvre de Chen Zhongshi, Bailu Yuan (cerf blanc-plateau): Au pays du cerf blanc. Le même procédé est constaté dans les titres de Zixingche zhi ge (bicyclette-DE-chant) de Su Tong, et de Xunzhao Shanghai (chercher-Shanghai) de Wang Anyi. Ils deviennent ainsi À bicyclette et À la recherche de Shanghai. La préposition peut être dans selon le nom de lieu qui suit, comme Dans l'empire des ténèbres alors que Chenlun de Shengdian (sombrer-DE-sanctuaire) de Liao Yiwu n'a pas de préposition. En a été ajouté dans En un mot comme en mille de Liu Zhenyun, alors que Yi ju ding yiwan $j u$, structure phrastique, signifie littéralement «une phrase en vaut dix mille».

Il faut souligner que par rapport au français, la distinction des catégories en chinois reste encore plus floue. Par exemple, Yun de Diao Dou, remplacé par Nid de coucou, peut signifier «être enceinte, grossesse». Le mot yun est à la fois nominal et verbal. Mais dans notre étude sur la recatégorisation, nous avons pris en compte uniquement les cas où le mot appartient à une seule catégorie grammaticale. 
Ces transformations sont, à notre avis, toutes bien réussies, car le sens des titres originaux reste inchangé, et les nouveaux titres correspondent mieux à l'usage de la langue française.

\subsection{Modulation}

La modulation est un procédé qui « consiste à restaurer un énoncé du texte d'arrivée en faisant intervenir un changement de point de vue ou d'éclairage par rapport à la formulation originale, ce qui se produit, notamment, quand on emploie la partie pour le tout, l'abstrait pour le concret, l'actif pour le passif» (Delisle et al. 1999: 54). Certains titres traduits restent dans le même esprit que l'original, mais se reformulent différemment. Quatre titres ont recours à cette méthode. La modulation donne un meilleur effet, un effet intensifié, tels que Tibet sans retour (Qu le Xizang, aller-LETibet) de Wang Chao et Une vie de silence (Meiyou yuyan de shenghuo, ne pas avoirlangue-DE-vie) de Dong Xi. Il est évident que qu le "être allé» et meiyou yuyan "il n'y a pas de langue» ne signifient pas forcément «sans retour» et «silence», mais ces deux nouvelles formulations semblent beaucoup plus originales, plus frappantes et plus riches en imagination.

Un seul cas nous rend un peu perplexe: Oublier Chengdu (Chengdu, jinye qing jiang wo yiwang, Chengdu-ce soir-prier-prép.-moi-oublier) de Murong Xuecun. Il reste discutable: le titre en chinois signifie «Chengdu, veuillez m'oublier cette nuit / oubliez-moi cette nuit», alors que le titre en français inverse le sujet et l'objet. Nous avons du mal à comprendre la raison pour laquelle le traducteur a fait une telle inversion. Il est certainement plus plausible de procéder à une traduction littérale: "Chengdu, laissez-moi seule cette nuit» ou "Chengdu, oubliez-moi cette nuit». D'ailleurs, c'est ce que la version anglaise a adopté: Chengdu, Leave Me Alone Tonight. Cette dernière permet probablement d'éviter le risque d'erreurs d'interprétation, et de conserver la particularité et la poétique du titre original.

\section{Explicitation}

L'explicitation consiste à «introduire dans le texte d'arrivée, pour plus de clarté ou en raison de contraintes imposées par la langue d'arrivée, des précisions sémantiques non formulées dans le texte de départ, mais qui se dégagent du contexte cognitif ou de la situation décrite» (Delisle et al. 1999: 37). Le traducteur peut ajouter des éléments culturels ou d'autres informations dans le titre traduit dans le but de rendre ce dernier plus explicite et plus accessible aux lecteurs francophones. Dans notre étude, 25 titres découlent de ce procédé. Les éléments explicités peuvent être un mot, une indication culturelle ou bien une information concernant le genre ou la forme de l'œuvre, qui sont nécessaires (voir Tableau IV).

TABLEAU IV

Répartition des titres traduits par ajout

\begin{tabular}{|l|l|c|}
\hline \multicolumn{2}{|l|}{ Nombre et pourcentage par rapport aux 237 titres } & $25(11 \%)$ \\
\hline \multirow{2}{*}{ Méthodes } & Explicitation d'éléments culturels & $7(28 \%)$ \\
\cline { 2 - 3 } & Explicitation d'autres informations & $18(72 \%)$ \\
\hline
\end{tabular}




\subsection{Explicitation d'éléments culturels nécessaires}

Pour faciliter la compréhension ou pour éveiller rapidement l'intérêt des lecteurs, afin que ces derniers y trouvent un écho immédiat, le traducteur juge nécessaire d'ajouter certains termes dans le titre traduit. Sur 25 titres traduits, 7 appartiennent à cette catégorie, soit $28 \%$. Er Ma (deux-Ma), de Lao She, raconte l'histoire de Monsieur Ma qui, en vue d'hériter du patrimoine de son frère aîné à Londres, fait un long voyage en bateau avec son fils. Arrivant à destination, lui et son fils connaissent chacun une aventure amoureuse. Le titre chinois est bref, alors que le titre français, Messieurs $M a$, père et fils, fait connaître $M a$ comme patronyme chinois, et précise que Er Ma sont père et fils. Le titre du majestueux roman Fengru feitun de Mo Yan est traduit par Beaux seins, belles fesses, auquel s'ajoute les enfants de la famille Shangguan. La partie traduite littéralement a provoqué des discussions dans les milieux de la traduction. L'expression figée Fengru feitun, tout à fait positive dans la langue chinoise, véhicule un éloge envers une femme et une mère bien en chair. Or, cette information culturelle disparaît dans la traduction littérale Beaux seins, belles fesses, qui ne donne pas forcément lieu à une suggestion similaire. Au contraire, elle risque d'être plus ou moins soupçonnée d'être un roman érotique. Le traducteur racontait dans une conférence donnée à l'Université de Genève en 2012 qu'il n'était pas satisfait du résultat, étant tout à fait conscient du malentendu que sa traduction risquait d'induire en étant provocante. La version anglaise traduite par Howard Goldblatt, Big Breasts and Wide Hips, laisse également à désirer. Prenons encore un exemple. Si le terme «sex» s'est ajouté au titre Xiongmao (panda), de Mian Mian, devenant Panda sex, cela tient étroitement au contenu du roman ayant pour thème majeur la description de la vie sexuelle de jeunes Chinois contemporains. Bien entendu, il n'est pas exclu que l'idée de cette explicitation vienne de l'éditeur et vise à augmenter la vente.

Yijiusanqi nian de aiqing (1937-année-DE-amour) de Ye Zhaoyan raconte une histoire d'amour en 1937 à Nankin. Puisque durant cette année et dans cette ville s'est produit le massacre de Nankin, ceci a provoqué une stupéfaction générale en Chine comme à l'étranger. Une histoire qui s'est déroulée dans un tel contexte manifeste l'importance du lieu Nankin. Avec cet ajout, le titre traduit, Nankin 1937, une histoire d'amour, rend immédiatement l'histoire extraordinaire.

\subsection{Explicitation d'autres informations complémentaires}

Il arrive que le traducteur ajoute quelques informations complémentaires concernant l'oeuvre, telles que le genre littéraire, l'appartenance à une série ou à une collection. Nous constatons que Shanhaijing zhuan (Shanghaijing-biographie) de Gao Xingjian devient Chronique du classique des mers et des monts: tragicomédie divine en trois actes, dont la partie ajoutée souligne la caractéristique théâtrale de cette pièce (tragicomédie divine en trois actes). Étant donné que Crime de sang (Xue zhi zui, sangDE-crime) de He Jiahong fait partie d'une série d'enquêtes pénales menées par le protagoniste de l'histoire, l'avocat Hong Jun, La Deuxième enquête de l'avocat Hong s'est logiquement ajouté au titre français.

Lorsqu'il s'agit d'un recueil de nouvelles ou de poèmes, une pratique assez courante consiste à extraire le titre d'une ouvre importante ou connue pour en faire le 
titre du recueil. Ensuite, on y ajoute des indications telles que «et autres nouvelles» ou «et autres poèmes». Parmi les 237 titres, 18 sont ainsi traduits. Par exemple, le recueil de textes en prose $Y u$ de ziwei (pluie-DE-goût) de Shi Zhecun regroupe de nombreuses nouvelles et proses. Le traducteur a choisi une des nouvelles du même nom comme titre, Le goût de la pluie, et y ajoute comme indication nouvelles et prose de circonstance.

\section{Implicitation}

À l'inverse de l'explicitation, l'implicitation est le «résultat d'une économie qu'on obtient en ne reformulant pas explicitement dans le texte d'arrivée des éléments d'information du texte de départ quand ils ressortent de façon évidente du contexte ou de la situation décrite» (Delisle et al. 1999: 44). Le traducteur peut enlever certains éléments du titre original, afin que le titre traduit soit plus vif et concis ou plus adapté aux habitudes du lectorat français. Sur les 237 titres, 15 sont traduits au moyen de l'implicitation, soit $6 \%$. Deux méthodes sont employées pour réduire le titre original: le raccourcissement et la suppression du verbe (voir Tableau V ci-dessous).

TABLEAU V

Répartition des titres rendus par implicitation

\begin{tabular}{|l|l|c|}
\hline Nombre et pourcentage par rapport aux 237 titres & $15(6 \%)$ \\
\hline \multirow{2}{*}{ Méthodes } & Suppression de certaines parties & $10(67 \%)$ \\
\cline { 2 - 3 } & Suppression du verbe & $5(33 \%)$ \\
\hline
\end{tabular}

\subsection{Supression de certaines parties}

Certains titres chinois contiennent des informations que le traducteur juge peu importantes, voire dérangeantes. Si on les garde, elles risquent de gêner la lecture ou la compréhension, au point de perdre leur fonction en tant que clé de l'œuvre. Dans ce cas-là, le traducteur préfère les supprimer et saisir l'essentiel. Pour Yuanliang wo hong chen diandao (pardonner-moi-rouge-poussière-inverser) de Murong Xuecun, le titre est réduit en Danse dans la poussière rouge. Ce dernier correspond plus ou moins à hongchen diandao (auquel le mot danse traduit l'idée de diandao). Les deux premiers mots, yuanliang wo (pardonnez-moi), sont supprimés. Dans L'énigme de la pierre Eil-de-Dragon (Rensheng wuqu-Longyan Shi zhi mi, vie-zone néfaste-Longyan-pierre-DE-énigme) de He Jiahong, la première partie, Rensheng wuqu (Les zones néfastes de la vie) a été supprimée, et seule la seconde partie est gardée. Meng de jieshi (rêve-DE-expliquer) devient Rêves, où le traducteur a laissé jieshi (expliquer) pour rendre le titre plus concis et plus attractif. Pour nous, l'exemple le mieux réussi est Début fatal (Zai wode kaishi shi wode jieshu, à-mon- début-être-mon-finir) de Fang Fang. Par rapport à la traduction littérale (Mon début est ma fin), nous trouvons Début fatal merveilleux, car en seulement deux mots, le traducteur réussit à exprimer de façon parfaite l'essence d'un début mortel.

La suppression la plus fréquente vise le verbe du titre. 


\subsection{Suppression $d u$ verbe}

Comme nous l'avons vu plus haut (voir 2.2.2.), de nombreuses structures verbales sont transformées en structures nominales. Dans 5 cas, le traducteur a tout simplement supprimé le verbe que contient le titre original. Dans Une fille pour mes 18 ans (Shiba sui gei wo yige guniang, 18-an-donner-une-fille) de Feng Tang, Sur la route à 18 ans (Shiba sui chumen yuan xing, 18-an-sortir-loin-voyager) de Yu Hua, les verbes dans ces titres originaux, gei (donner) et chumen (partir), ont tous disparu. Le roman Tigre et dragon (Wo hu cang long, coucher-tigre-cacher-dragon) de Wang Dulu, et surtout le film du même nom, sont devenus très populaires. C'est aussi probablement en partie grâce à la suppression des deux verbes, wo (coucher) et cang (cacher), que le titre français est devenu court et facile à mémoriser. La suppression la plus extraordinaire revient à Épouses et concubines (Qi qie cheng qun, épouse-concubine-formertroupe) de Su Tong. Au moyen d'une forme grammaticale simple, le pluriel, le traducteur a réussi à exprimer le sens du verbe cheng qun, littéralement «former une troupe», faisant ainsi preuve d'imagination et d'originalité. Le contraire, où l'on ajoute un verbe dans le titre traduit, existe aussi, mais c'est rarissime. L'exemple le plus illustre est Wode diwang shengya (mon-empereur- carrière), littéralement $M a$ carrière en tant qu'empereur de Chine, de Su Tong, rendu par Je suis l'empereur de Chine. Le mélange de fierté et d'ironie induit par ce titre est tout simplement extraordinaire sur le plan de la traduction.

Si nous nous penchons sur un élément particulier des titres, soit le nom propre, nous constatons que la suppression est aussi une méthode courante. Parmi les 237 titres en chinois, 57 renferment au moins un nom propre, soit $24 \%$ de la totalité. Lorsque ces titres sont rendus en français, 38 conservent le même nom propre par transcription en pinyin; 22 noms propres sont supprimés ou transformés en nom commun; 14 nouveaux noms propres sont ajoutés par le traducteur; 2 nouveaux noms propres ont remplacé les 2 noms propres du titre original. Au total, 48 titres en français contiennent un nom propre, soit $20 \%$.

\subsection{Plusieurs procédés combinés}

Nous venons d'analyser l'un après l'autre les quatre procédés envisagés. Mais la traduction d'un titre peut combiner plusieurs procédés. Si l'on compare la longueur des deux titres, la traduction Début fatal de Fang Fang et le mot-à-mot, Zai wode kaishi shi wode jieshu (à-mon-début-être-mon-finir), on constate que dans le premier, trois éléments, le verbe shi (voir 4.2.), la préposition zai et le pronom possessif wode ont été supprimés. La structure phrastique de l'original devient une structure nominale. Du coup, le titre en français est considérablement raccourci par rapport à l'original (deux mots au lieu de six), donc bref et frappant. L'exemple de Shu yu lin tong zai (arbre-forêt-ensemble-exister) de Liu Xinwu est aussi parlant. Rendu par L'arbre et la forêt: destins croisés, on peut remarquer une modification lexicale, de tong zai (ensemble-exister) en destins croisés, une transformation de la structure verbale en structure nominale et un ajout de la ponctuation (les deux points). Shui ru dadi (eauallaiter-terre) de Fan Wen est rendu par Une terre de lait et de miel. Le traducteur a opéré une transformation du verbe $r u$ en nom lait, un ajout du mot miel, et une suppression du mot eau. Même dans la traduction dite littérale, on peut trouver 
certains changements qui entraînent des nuances. Zui (crime) de Diao Dou, traduit par La faute, était à la limite d'être considéré comme une traduction littérale, car les deux notions, zui en chinois et faute en français, ne couvrent pas exactement le même contenu.

Nous constatons également que l'emprunt de mots anglais est rare. Cela nous semble à la fois appréciable et réjouissant, et doit servir de modèle à d'autres langues pour qu'elles défendent leurs puretés respectives. À l'heure actuelle, où la langue de Shakespeare prédomine sur la scène internationale, ses mots s'introduisent de manière irrésistible dans les prétendues «petites» langues occidentales. L'anglicisme est, malheureusement, un phénomène mondialisé ${ }^{10}$. Dans ce contexte, nos quelques titres contenant des mots anglais sont tout à fait anecdotiques. De plus, plusieurs de ces mots proviennent d'une traduction phonétique de l'anglais. La version française les a simplement remis en anglais. Par exemple, xiu dans Shenghuo xiu (vie-show) est remis à show (Le show de la vie de Chi Li), wutuobang dans Gouwu wutuobang (achatutopie) à utopia (Shopping utopia de Hu Fang), ou encore Yinggelishi à English de Wang Gang. Notons que le mot shopping est accepté depuis longtemps par le Larousse. Ou encore, la forme et le sens des mots en français et en anglais sont similaires, comme "utopia» et "utopie», «sex» et «sexe» (dans Panda sex de Mian Mian).

Deux autres cas sont discutables. Xiongdi de Yu Hua, a aussi pris sa traduction anglaise, Brothers. Pourquoi pas «Frères»? Les frères en question sont à la fois vrais et faux, mais «frère», identique à «brother», pourrait aussi avoir un double sens. Peut-être tout simplement parce que le titre Brothers figure déjà sur la couverture de l'original. Se jie (couleur-prendre garde) de Zhang Ailing est rendu par Lust, caution: amour, luxure, trahison. Ce dernier contient le titre en anglais et y ajoute la partie en français. L'auteur, résidant pendant de nombreuses années aux États-Unis, est une écrivaine chinoise connue des lecteurs occidentaux. Ses nombreux ouvrages ont été traduits en anglais. Mais est-ce que cela peut jouer en faveur de la reprise des titres en anglais pour une traduction en français? Quelle est la motivation qui incite les traducteurs à garder les titres anglais? Profiter de l'aura de l'anglais ou de la version anglaise? À signaler que la version française de Se jie est parue en 2009, alors que le film du réalisateur Li Ang portant le même titre venait de remporter un grand succès au cinéma.

\section{Conclusion}

Nous avons vu à travers notre étude que la traduction de titres littéraires n'est pas seulement un travail de restitution du sens des mots qui composent le titre. Quelle que soit sa forme, c'est une image forte et symbolique qui doit représenter l'œuvre avec le plus de pertinence possible. Les différents procédés mis en application montrent que chaque traducteur examine en profondeur les possibilités éventuelles pour mieux rendre le titre de l'œuvre qu'il traduit. Il réfléchit aux multiples facettes liées à un titre littéraire: son effet, sa précision, sa lisibilité et sa réception, sa connotation, son symbolisme. En un mot, sa complexité. Il explore toute sa portée avant de prendre une décision finale.

Chaque titre est un cas particulier. Cependant, notre analyse nous permet de découvrir quelques règles et conventions régissant la traduction de titres du chinois au français: 1) les traducteurs s'efforcent, autant que possible, d'être fidèles aux titres 
d'origine en les traduisant plus ou moins littéralement; presque la moitié des titres traduits ont respecté le sens et la forme de l'original, c'est-à-dire l'intention de l'auteur; 2) selon les habitudes de la langue française, les titres de nature verbale en chinois sont souvent rendus en nominale en français; le traducteur soit supprime le verbe, soit transforme le verbal en nominal; 3) les traducteurs choisissent souvent intentionnellement un titre nominal sans article, contrairement à l'usage du français qui associe souvent un article à un nom; un nom sans article, assez inhabituel, peut produire un effet imaginaire, exotique et surprenant; 4) lorsqu'une traduction littérale apparaît insuffisante ou inappropriée, le traducteur se permet de modifier le titre; il peut soit supprimer certains éléments superflus, soit en ajouter d'autres apportant des informations jugées importantes, voire indispensables; 5) pour une meilleure adaptation culturelle ou des considérations commerciales, le traducteur s'éloigne du titre original; il va jusqu'à en inventer un ; 6) un effort particulier est perçu dans la combinaison d'une ressemblance de forme et d'esprit entre le titre original et le titre traduit. Les procédés relevés par notre recherche sont dans la même ligne que les solutions obtenues par l'étude de Malingret (1998: 400-405) dans la traduction des titres de l'espagnol en français: non-traduction, traduction littérale, adaptation, substitution. Bien entendu la non-traduction, complète ou partielle, possible entre deux langues proches comme le français et l'espagnol, s'avère impossible entre des langues éloignées telles que le chinois et le français.

Nous avons aussi vérifié l'hypothèse selon laquelle tel traducteur applique plutôt une méthode plutôt qu'une autre, reconnaissant que la traduction, surtout littéraire, est une activité créatrice et que, par conséquent, chacun peut avoir son style et ses préférences. Après investigation, nous constatons que la tendance individuelle ne joue pas un rôle suffisamment significatif dans le choix des procédés. Étant donné que nous nous trouvons dans un environnement social où priment les avantages économiques, la modification et le choix des titres n'excluent pas une intervention énergique de la part des rédacteurs et des éditeurs. Mais notre analyse montre qu'à l'évidence les traducteurs littéraires attachent plus d’importance à la littéralité qu'au succès commercial. Bien que la poursuite de ce dernier soit toujours présente, les traducteurs disposent tout de même d'une liberté et d'une marge tout à fait honorables.

\section{NOTES}

1. Roy (2008: 47) a mentionné une enquête menée auprès des lycéens québécois au Canada: $37,6 \%$ d'entre eux estiment que les titres, exerçant une grande influence sur leur lecture, sont «très importants".

2. Nous nous basons principalement sur le recensement des œuvres contemporaines chinoises traduites en français par Pino (2014), sans comptabiliser les traductions publiées dans les revues, les actes de colloque et les anthologies.

3. Ces cinq titres sont Yequan husheng 野犬呼声, Yequan feisheng 野犬吠声, Yexing de husheng 野性 的呼声 Yexing de huhuan 野性的呼唤, Huangye de huhuan 荒野的呼唤. Ces variations apparemment mineures peuvent correspondre à des différences relativement importantes dans la compréhension de l'œuvre elle-même.

4. Pour faciliter la lecture, les titres et les auteurs sont transcrits uniquement en pinyin. Veuillez consulter le détail des œuvres citées dans l'annexe à la fin de l'article. Les 237 titres sont classés selon l'ordre aphabétique des noms des auteurs.

5. Quant à Howard Goldblatt, traducteur américain de la version anglaise, il a préféré Daughter of the River, puisque l'auteur Hong Ying est d'origine de Chongqing, et que l'histoire se passe justement le long du Yangtsé près de cette ville. 
6. Yu (2008: 72-74) montre qu'il existe quatre sortes d'erreurs dans la traduction des titres d'œuvres littéraires: donner libre cours à sa créativité (ziyou fahui 自由发挥), tendre à être vulgaire (meisu qingxiang 媚俗倾向), se couper du contexte (tuoli yujing 脱离语境) et être trop littérale (guoyu zhibai 过于直白). Bien qu'il ait consacré ses études à la traduction de l'anglais vers le chinois, le résultat de ses études convient également à la traduction entre d'autres langues.

7. Nous trouvons le même phénomène dans la traduction du chinois vers l'anglais. Yang (1996: 40) a examiné les titres de Ying yi tangren jueju bai shou (英译唐人绝句百首, Cent quatrains en vers de cinq ou sept pieds de la dynastie des Tang traduits en anglais), compilé par Lü Shuxiang 吕叔湘 (Éditions du Peuple du Hunan, 1980): seuls 40 titres sont traduits littéralement, alors que les 60 autres sont substitués par un nouveau titre.

8. Notre traduction.

9. Le traitement des noms propres dans la traduction des titres littéraires fera l'objet d'une autre étude.

10. Selon Le Figaro du 8 septembre 2010 (Aissaoui et Dargent 2010), les titres anglais s'emploient de plus en plus dans la littérature française, et cette situation n'est pas forcément «déplaisante».

\section{RÉFÉRENCES}

Aissaoui, Mohammed et Dargent, Françoise (2010): À la recherche du titre idéal. Le Figaro. Consulté le 20 septembre 2014, <http://www.lefigaro.fr/livres/2010/09/08/0300520100908artfig00545--la-recherche-du-titre-ideal.php >.

Billeter, Jean-François (2014): Trois essais sur la traduction. Paris: Allia.

Delisle, Jean, Lee-Jahnke, Hannelore, Cormier, Monique (1999): Terminologie de la traduction. Amsterdam / Philadelphie: John Benjamins Publishing Company.

Eco, Umberto (1979/1985): Lector in fabula: le rôle du traducteur, ou, La coopération interprétative dans les textes narratifs. (Traduit de l'italien par Myriem Bouzaher). Paris: Grasset.

Grevisse, Maurice (1993): Le bon usage (13 éd.). Paris: Duculot.

Malingret, Laurence (1998): Les titres en traduction. In: Teresa García-Sabell, Dolores Olivares, Annick Boiléve-Guerlet, Manuel García, dir. Les chemins du texte. 2:396-407.

Pino, Anne (2014): Bibliographie générale des œuvres littéraires modernes d'expression chinoise traduites en français. Paris: You Feng.

Roy, Max (2008): Du titre littéraire et de ses effets de lecture. In: Protée. 36(3):47-56.

SHEN, Qingli 申清丽 (2013): Qianxi wenxue zuopin biaoti yiming 浅析文学作品标题译名 [Analyse de la traduction des titres littéraires]. Zuojia zazhi 作家杂志. 5:199-200.

WANG, Mingyuan 王明元 (1994): Manyi waiguo wenxue mingzhu timing de hanyi 漫议外国文 学名著题名的汉译 [Discussion sur la traduction chinoise des titres des classiques étrangers]. Zhongguo fanyi 中国翻译. 6:23-27.

YANG, Yuangang 杨元刚 (1996): Mantan biaoti de fanyi --- Jian yu Lu Yunzhong jiaoshou shangcuo 漫谈标题的翻译——兼与卢允中教授商磋 [Discussion sur la traduction des titres — Et négociations avec Prof. Lu Yunzhong]. Shandong waiyu jiaoxue 山东外语教学. 1:3943.

Yu, Jianhua 虞建华 (2008): Wenxue zuopin biaoti de fanyi: tezheng yu wuqu 文学作品标题的 翻译: 特征与误区 [La traduction des titres littéraires: caractéristiques et malentendus]. Waiguoyu 外国语. 31/1:68-74.

ZHANG, Jinting 张金廷 (2001): Shuming fanyi zhong de du书名翻译中的度 [Le degré dans la traduction des titres de livre]. Shandong shifan daxue 山东师范大学. 2:70-72. 


\section{ANNEXE}

237 œuvres littéraires chinoises traduites en français publiées par éditeurs français entre 2000 et 2014 listées selon l'ordre alphabétique de l'auteur (sans tenir compte de celles publiées dans des revues, actes de colloques, anthologies)

\begin{tabular}{|c|c|c|c|c|c|}
\hline Auteur & Titre chinois & Titre français & Traducteur & Éditeur & Parution \\
\hline Bei Dao 北岛 (1949-) & $\begin{array}{l}\text { 零度以上的风景 } \\
\text { 线 }\end{array}$ & $\begin{array}{l}\text { Paysage au-dessus de } \\
\text { zéro }\end{array}$ & $\begin{array}{l}\text { Chantal } \\
\text { Chen-Andro }\end{array}$ & Circé & 2004 \\
\hline Bi Feiyu 毕飞宇 (1964-) & 青衣 & Lopéra de la lune & Claude Payen & Picquier & 2003 \\
\hline Bi Feiyu 毕飞宇 (1964-) & 玉米 & Trois sœurs & Claude Payen & Picquier & 2004 \\
\hline Bi Feiyu 毕飞宇 (1964-) & 雨天的棉花糖 & $\begin{array}{l}\text { De la barbe à papa } \\
\text { un jour de pluie }\end{array}$ & Isabelle Rabut & Actes Sud & 2004 \\
\hline Bi Feiyu 毕飞宇 (1964-) & 上海往事 & $\begin{array}{l}\text { Les triades de } \\
\text { Shanghai }\end{array}$ & Claude Payen & Picquier & 2007 \\
\hline Bi Feiyu 毕飞宇 (1964-) & 平原 & La plaine & Claude Payen & Picquier & 2009 \\
\hline Bi Feiyu 毕飞宇 (1964-) & 推拿 & Les aveugles & $\begin{array}{l}\text { Emmanuelle } \\
\text { Péchenart }\end{array}$ & Picquier & 2011 \\
\hline Can Xue 残雪 (1953-) & 黄泥街 & $\begin{array}{l}\text { La rue de la boue } \\
\text { jaune }\end{array}$ & $\begin{array}{l}\text { Geneviève } \\
\text { Imbot-Bichet }\end{array}$ & $\begin{array}{l}\text { Bleu de } \\
\text { Chine }\end{array}$ & 2001 \\
\hline $\begin{array}{l}\text { Cao Naiqian 曹乃谦 } \\
\text { (1949-) }\end{array}$ & $\begin{array}{l}\text { 到黑夜想你没办 } \\
\text { 法 }\end{array}$ & $\begin{array}{l}\text { La nuit quand tu me } \\
\text { manques, j’peux rien } \\
\text { faire: panorama du } \\
\text { village des Wen }\end{array}$ & $\begin{array}{l}\text { Françoise } \\
\text { Bottéro, Fu Jie }\end{array}$ & Gallimard & 2011 \\
\hline $\begin{array}{l}\text { Cao Wenxuan 曹文轩 } \\
(1954-)\end{array}$ & 青铜葵花 & Bronze et Tournesol & $\begin{array}{l}\text { Brigitte } \\
\text { Guilbaud }\end{array}$ & Picquier & 2010 \\
\hline $\begin{array}{l}\text { Chen Yingzhen 陈映真 } \\
\text { (1936-) }\end{array}$ & 将军族 & L'île verte & Anne Breuval & $\begin{array}{l}\text { Bleu de } \\
\text { Chine }\end{array}$ & 2000 \\
\hline $\begin{array}{l}\text { Chen Zhongshi陈忠实 } \\
\text { (1942-) }\end{array}$ & 白鹿原 & Au pays du cerf blanc & $\begin{array}{l}\text { Shao Baoqing, } \\
\text { Solange } \\
\text { Cruveillé }\end{array}$ & Seuil & 2012 \\
\hline Chi Li 池莉 (1957-) & 你以为你是谁 & $\begin{array}{l}\text { Pour qui te } \\
\text { prends-tu? }\end{array}$ & Hervé Denès & Actes Sud & 2000 \\
\hline Chi Li 池莉 (1957-) & 预谋杀人 & Préméditation & $\begin{array}{l}\text { Angel Pino, } \\
\text { Shao Baoqing }\end{array}$ & Actes Sud & 2002 \\
\hline Chi Li 池莉 (1957-) & 你是一条河 & Tu es une rivière & $\begin{array}{l}\text { Angel Pino, } \\
\text { Isabelle Rabut }\end{array}$ & Actes Sud & 2004 \\
\hline Chi Li 池莉 (1957-) & 太阳出世 & Soleil levant & Angel Pino & Actes Sud & 2005 \\
\hline Chi Li 池莉 (1957-) & 有了快感你就喊 & $\begin{array}{l}\text { Un homme bien sous } \\
\text { tous rapports }\end{array}$ & Hervé Denès & Actes Sud & 2006 \\
\hline Chi Li 池莉 (1957-) & 看麦娘 & $\begin{array}{l}\text { Les sentinelles des } \\
\text { blés }\end{array}$ & $\begin{array}{l}\text { Shao Baoqing, } \\
\text { Angel Pino }\end{array}$ & Actes Sud & 2008 \\
\hline Chi Li 池莉 (1957-) & 生活秀 & Le show de la vie & Hervé Denès & Actes Sud & 2011 \\
\hline Chi Zijian 迟子建 (1964-) & $\begin{array}{l}\text { 世界上所有的夜 } \\
\text { 晚 }\end{array}$ & $\begin{array}{l}\text { Toutes les nuits du } \\
\text { monde }\end{array}$ & $\begin{array}{l}\text { Stéphane } \\
\text { Lévêque, } \\
\text { Yvonne André }\end{array}$ & Picquier & 2013 \\
\hline Cui Zi’n 崔子恩 & 桃色嘴唇 & Lèvres pêche & Sylvie Gentil & Gallimard & 2010 \\
\hline Dai Lai 戴来 (1972-) & 对面有人 & L'insecte sur la toile & $\begin{array}{l}\text { Véronique } \\
\text { Chevaleyre }\end{array}$ & $\begin{array}{l}\text { Bleu de } \\
\text { Chine }\end{array}$ & 2003 \\
\hline Diao Dou フ斗 (1960-) & 孪生 & Jumeaux & $\begin{array}{l}\text { Anne } \\
\text { Thiollier, } \\
\text { Catherine Lan }\end{array}$ & $\begin{array}{l}\text { Bleu de } \\
\text { Chine }\end{array}$ & 2001 \\
\hline Diao Dou フ斗 (1960-) & 解决 & Solutions & $\begin{array}{l}\text { Anne } \\
\text { Thiollier, } \\
\text { Catherine Lan }\end{array}$ & $\begin{array}{l}\text { Bleu de } \\
\text { Chine }\end{array}$ & 2002 \\
\hline Diao Dou フ斗 (1960-) & 孕 & Nid de coucou & $\begin{array}{l}\text { Véronique } \\
\text { Jacquet- } \\
\text { Woillez }\end{array}$ & $\begin{array}{l}\text { Bleu de } \\
\text { Chine }\end{array}$ & 2003 \\
\hline Diao Dou フ斗 (1960-) & 罪 & La faute & $\begin{array}{l}\text { Véronique } \\
\text { Jacquet- } \\
\text { Woillez }\end{array}$ & $\begin{array}{l}\text { Bleu de } \\
\text { Chine }\end{array}$ & 2004 \\
\hline Diao Dou フ斗 (1960-) & 梦的解释 & Rêves & Prune Cornet & $\begin{array}{l}\text { Bleu de } \\
\text { Chine }\end{array}$ & 2006 \\
\hline
\end{tabular}




\begin{tabular}{|c|c|c|c|c|c|}
\hline Auteur & Titre chinois & Titre français & Traducteur & Éditeur & Parution \\
\hline Dong Xi 东西 (1966-) & 救命 & Sauver une vie & $\begin{array}{l}\text { Amélie } \\
\text { Manon }\end{array}$ & L'Aube & 2007 \\
\hline Dong Xi 东西 (1966-) & 没有语言的生活 & $\begin{array}{l}\text { Une vie de silence, et } \\
\text { autres nouvelles }\end{array}$ & Isild Darras & L'Aube & 2010 \\
\hline Dong Xi 东西 (1966-) & 把嘴角挂在耳边 & $\begin{array}{l}\text { Accrocher les coins } \\
\text { de la bouche au bord } \\
\text { des oreilles }\end{array}$ & Isild Darras & L'Aube & 2013 \\
\hline Dong Xi 东西 (1966-) & $\begin{array}{l}\text { 你不知道她有多 } \\
\text { 美 }\end{array}$ & $\begin{array}{l}\text { Tu ne sais pas } \\
\text { combien elle est belle }\end{array}$ & Isild Darras & L'Aube & 2013 \\
\hline Duo Duo 多多 (1951-) & 桑那在尔的诗 & $\begin{array}{l}\text { Poèmes de } \\
\text { Saint-Nazaire }\end{array}$ & $\begin{array}{l}\text { Chantal } \\
\text { Chen-Andro }\end{array}$ & MEET & 2008 \\
\hline Fan Wen 范稳 (1962-) & 水乳大地 & $\begin{array}{l}\text { Une terre de lait et de } \\
\text { miel }\end{array}$ & $\begin{array}{l}\text { Stéphane } \\
\text { Lévêque }\end{array}$ & Picquier & 2013 \\
\hline Fang Fang 方方 (1955-) & $\begin{array}{l}\text { 在我的开始是我 } \\
\text { 的结束 }\end{array}$ & Début fatal & $\begin{array}{l}\text { Geneviève } \\
\text { Imbot-Bichet }\end{array}$ & Stock & 2001 \\
\hline $\begin{array}{l}\text { Feng Zikai 丰子恺 } \\
\text { (1898-1975) }\end{array}$ & 云霓 & Couleur de nuage & $\begin{array}{l}\text { Marie } \\
\text { Laureillard- } \\
\text { Wendland } \\
\end{array}$ & Gallimard & 2010 \\
\hline Feng Hua 冯华 (1972-) & 如影随形 & $\begin{array}{l}\text { Seul demeure son } \\
\text { parfum }\end{array}$ & $\begin{array}{l}\text { Li Hong, } \\
\text { Gilles } \\
\text { Moraton }\end{array}$ & Picquier & 2009 \\
\hline Feng Jicai 冯瞕才 (1942-) & 一百个人的十年 & $\begin{array}{l}\text { Lempire de l'absurde, } \\
\text { ou Dix ans de la vie } \\
\text { de gens ordinaires }\end{array}$ & $\begin{array}{l}\text { Marie-France } \\
\text { de Mirbeck, } \\
\text { Etiennette } \\
\text { Nodot }\end{array}$ & $\begin{array}{l}\text { Bleu de } \\
\text { Chine }\end{array}$ & 2001 \\
\hline Feng Jicai 冯瞕才 (1942-) & 俗世奇人 & $\begin{array}{l}\text { Le petit lettré de } \\
\text { Tianjin }\end{array}$ & $\begin{array}{l}\text { Marie-France } \\
\text { de Mirbeck }\end{array}$ & $\begin{array}{l}\text { Bleu de } \\
\text { Chine }\end{array}$ & 2002 \\
\hline Feng Jicai 冯䫍才 (1942-) & 传奇 & Personnages & $\begin{array}{l}\text { Jacques } \\
\text { Meunier }\end{array}$ & You Feng & 2008 \\
\hline Feng Jicai 冯瀷才 (1942-) & 抒情 & Sentiments & $\begin{array}{l}\text { Yang Fen, } \\
\text { Jacques } \\
\text { Meunier }\end{array}$ & $\begin{array}{l}\text { Bleu de } \\
\text { Chine }\end{array}$ & 2009 \\
\hline Feng Jicai 冯瞕才 (1942-) & 幽默 & Humour & $\begin{array}{l}\text { Yang Fen, } \\
\text { Jacques } \\
\text { Meunier }\end{array}$ & $\begin{array}{l}\text { Bleu de } \\
\text { Chine }\end{array}$ & 2010 \\
\hline Feng Tang 冯唐 (1971-) & 万物生长 & $\begin{array}{l}\text { Qiu, comme } \\
\text { l'automne }\end{array}$ & Sylvie Gentil & L'Olivier & 2007 \\
\hline Feng Tang 冯唐 (1971-) & $\begin{array}{l}\text { 十八岁给我一个 } \\
\text { 姑娘 }\end{array}$ & $\begin{array}{l}\text { Une fille pour mes } 18 \\
\text { ans }\end{array}$ & Sylvie Gentil & L'Olivier & 2009 \\
\hline $\begin{array}{l}\text { Gao Xingjian 高行健 } \\
(1940-)\end{array}$ & 一个人的圣经 & $\begin{array}{l}\text { Le livre d'un homme } \\
\text { seul }\end{array}$ & $\begin{array}{l}\text { Liliane } \\
\text { Dutrait, Noël } \\
\text { Dutrait }\end{array}$ & L'Aube & 2000 \\
\hline $\begin{array}{l}\text { Gao Xingjian 高行健 } \\
(1940-)\end{array}$ & 山海经传 & $\begin{array}{l}\text { Chronique du } \\
\text { classique des mers et } \\
\text { des monts: } \\
\text { tragicomédie divine } \\
\text { en trois actes }\end{array}$ & $\begin{array}{l}\text { Noël Dutrait, } \\
\text { Philippe Che }\end{array}$ & Seuil & 2013 \\
\hline $\begin{array}{l}\text { Gao Xingjian 高行健 } \\
(1940-)\end{array}$ & 逃亡 & La fuite & Julien Gelas & Lansman & 2013 \\
\hline Ge Fei 格非 (1964-) & 雨季的感受 & $\begin{array}{l}\text { Impressions à la } \\
\text { saison des pluies }\end{array}$ & $\begin{array}{l}\text { Xiaomin } \\
\text { Giafferi- } \\
\text { Huang, } \\
\text { Marie-Claude } \\
\text { Cantournet- } \\
\text { Jacquet }\end{array}$ & L'Aube & 2003 \\
\hline Ge Fei 格非 (1964-) & 俊瓜的诗篇 & Poèmes à l'idiot & $\begin{array}{l}\text { Xiaomin } \\
\text { Giafferi- } \\
\text { Huang }\end{array}$ & L'Aube & 2007 \\
\hline
\end{tabular}




\begin{tabular}{|c|c|c|c|c|c|}
\hline Auteur & Titre chinois & Titre français & Traducteur & Éditeur & Parution \\
\hline Ge Fei 格非 (1964-) & 蚌壳 & Coquillages & $\begin{array}{l}\text { Xiaomin } \\
\text { Giafferi- } \\
\text { Huang }\end{array}$ & L'Aube & 2008 \\
\hline Ge Fei 格非 (1964-) & 人面桃花 & $\begin{array}{l}\text { Une jeune fille au } \\
\text { teint de pêche }\end{array}$ & $\begin{array}{l}\text { Li et Bernard } \\
\text { Bourrit }\end{array}$ & $\begin{array}{l}\text { Gallimard, } \\
\text { Bleu de } \\
\text { Chine }\end{array}$ & 2012 \\
\hline $\begin{array}{l}\text { Gerileqimuge 格日勒其木 } \\
\text { 格 (1975-) }\end{array}$ & 黑烟 & Flamme & Patricia Batto & Picquier & 2011 \\
\hline Gu Long 古龙 (1937-1985) & 欢乐英雄 & $\begin{array}{l}\text { Les quatre brigands } \\
\text { du Huabei }\end{array}$ & $\begin{array}{l}\text { Christine } \\
\text { Corniot }\end{array}$ & Picquier & 2001 \\
\hline Gu Long 古龙 (1937-1985) & $\begin{array}{l}\text { 楚留香传奇: 血 } \\
\text { 海飘香 }\end{array}$ & $\begin{array}{l}\text { Les aventures de Chu } \\
\text { Liuxiang: un parfum } \\
\text { de pivoine sur la mer } \\
\text { écarlate }\end{array}$ & $\begin{array}{l}\text { François } \\
\text { Lagarde }\end{array}$ & You Feng & $\begin{array}{l}2009 \\
\text { vol } 1\end{array}$ \\
\hline Gu Long 古龙 (1937-1985) & $\begin{array}{l}\text { 楚留香传奇: 大 } \\
\text { 沙漠 }\end{array}$ & $\begin{array}{l}\text { Les aventures de Chu } \\
\text { Liuxiang: aventures } \\
\text { dans le désert de } \\
\text { Gobi }\end{array}$ & $\begin{array}{l}\text { François } \\
\text { Lagarde }\end{array}$ & You Feng & $\begin{array}{l}2010 \mathrm{vol} \\
2\end{array}$ \\
\hline $\begin{array}{l}\text { Guo Songfang 郭松芬 } \\
(1938-2005)\end{array}$ & 月音 & Récits de lune & $\begin{array}{l}\text { Marie } \\
\text { Laureillard }\end{array}$ & Zulma & 2007 \\
\hline $\begin{array}{l}\text { Guo XIaolu 郭小橹 } \\
(1973-)\end{array}$ & 我心中的石头镇 & La ville de pierre & Claude Payen & Picquier & 2004 \\
\hline $\begin{array}{l}\text { Guo Xuebo 郭雪波 } \\
(1948-)\end{array}$ & 沙狐 & Le renard du désert & Dong Chun & $\begin{array}{l}\text { Bleu de } \\
\text { Chine }\end{array}$ & 2001 \\
\hline Han Han 韩寒 (1982-) & 三重门 & Les trois portes & $\begin{array}{l}\text { Guan Jian, } \\
\text { Sylvie } \\
\text { Schneiter }\end{array}$ & Lattès & 2004 \\
\hline Han Han 韩寒 (1982-) & 中国博客 & Blog de Chine & Hervé Denès & $\begin{array}{l}\text { Gallimard, } \\
\text { Bleu de } \\
\text { Chine }\end{array}$ & 2012 \\
\hline Han Han 韩寒 (1982-) & $\begin{array}{l}\text { 1988: 我想和这 } \\
\text { 个世界谈谈 }\end{array}$ & $\begin{array}{l}\text { 1988: je voudrais } \\
\text { bien discuter avec le } \\
\text { monde }\end{array}$ & Hélène Arthus & $\begin{array}{l}\text { Gallimard, } \\
\text { Bleu de } \\
\text { Chine } \\
\end{array}$ & 2013 \\
\hline $\begin{array}{l}\text { Han Shaogong韩少功 } \\
\text { (1953-) }\end{array}$ & 山上的声音 & $\begin{array}{l}\text { Bruits dans les } \\
\text { montagnes, et autres } \\
\text { nouvelles }\end{array}$ & Anne Curien & Gallimard & 2000 \\
\hline He Jiahong 何家弘 (1953-) & 血之罪 & Crime de sang & $\begin{array}{l}\text { Marie-Claude } \\
\text { Cantournet- } \\
\text { Jacquet }\end{array}$ & L'Aube & 2003 \\
\hline He Jiahong 何家弘 (1953-) & $\begin{array}{l}\text { 人生误区一一龙 } \\
\text { 眼石之谜 }\end{array}$ & $\begin{array}{l}\text { Lénigme de la pierre } \\
\text { Oeil-de-Dragon }\end{array}$ & $\begin{array}{l}\text { Marie-Claude } \\
\text { Cantournet- } \\
\text { Jacquet, } \\
\text { Xiaomin } \\
\text { Giafferri- } \\
\text { Huang }\end{array}$ & L'Aube & 2004 \\
\hline He Jiahong 何家弘 (1953-) & 股市幕后的罪恶 & $\begin{array}{l}\text { Crimes et délits à la } \\
\text { Bourse de Pékin }\end{array}$ & $\begin{array}{l}\text { Marie-Claude } \\
\text { Cantournet- } \\
\text { Jacquet, } \\
\text { Xiaomin } \\
\text { Giafferri- } \\
\text { Huang }\end{array}$ & L'Aube & 2005 \\
\hline He Jiahong 何家弘 (1953-) & $\begin{array}{l}\text { 人生狭路--黑蝙 } \\
\text { 蝠白蝙蝠 }\end{array}$ & $\begin{array}{l}\text { Crime impuni aux } \\
\text { monts Wuyi }\end{array}$ & $\begin{array}{l}\text { Marie-Claude } \\
\text { Cantournet- } \\
\text { Jacquet }\end{array}$ & L'Aube & 2013 \\
\hline He Jiahong 何家弘 (1953-) & 神秘的古画 & $\begin{array}{l}\text { Le mystérieux tableau } \\
\text { ancien: la première } \\
\text { enquête de l'avocat } \\
\text { Hong }\end{array}$ & $\begin{array}{l}\text { Marie-Claude } \\
\text { Cantournet- } \\
\text { Jacquet, } \\
\text { Xiaomin } \\
\text { Giafferi- } \\
\text { Huang }\end{array}$ & L'Aube & 2013 \\
\hline
\end{tabular}




\begin{tabular}{|c|c|c|c|c|c|}
\hline Auteur & Titre chinois & Titre français & Traducteur & Éditeur & Parution \\
\hline Hong Ying 虹影 (1962-) & 饥饿的女儿 & Une fille de la faim & $\begin{array}{l}\text { Nathalie } \\
\text { Louisgrand }\end{array}$ & Seuil & 2000 \\
\hline Hong Ying 虹影 (1962-) & $\begin{array}{l}\text { 英国情人 } \\
(\text { 或K) }\end{array}$ & $\begin{array}{l}\text { Le livre des secrets de } \\
\text { l'alcôve }\end{array}$ & $\begin{array}{l}\text { Véronique } \\
\text { Jacquet- } \\
\text { Woillez }\end{array}$ & Seuil & 2003 \\
\hline Hu Fang 胡昉 (1970-) & 购物乌托邦 & Shopping utopia & $\begin{array}{l}\text { Caroline } \\
\text { Grillot }\end{array}$ & $\begin{array}{l}\text { Bleu de } \\
\text { Chine }\end{array}$ & 2003 \\
\hline $\begin{array}{l}\text { Huang Beijia 黄蓓佳 } \\
\text { (1955-) }\end{array}$ & 我要做个好孩子 & Lécole des vers à soie & $\begin{array}{l}\text { Patricia Batto, } \\
\text { Gao Tian Hua }\end{array}$ & Picquier & 2002 \\
\hline $\begin{array}{l}\text { Huang Beijia 黄蓓佳 } \\
(1955-)\end{array}$ & 这一瞬间如此辉 & $\begin{array}{l}\text { Ephémère beauté des } \\
\text { cerisiers en fleurs }\end{array}$ & $\begin{array}{l}\text { Philippe } \\
\text { Denizet }\end{array}$ & You Feng & 2005 \\
\hline $\begin{array}{l}\text { Huang Beijia 黄蓓佳 } \\
\text { (1955-) }\end{array}$ & 亲亲我的妈妈 & $\begin{array}{l}\text { Comment j'ai } \\
\text { apprivoisé ma mère }\end{array}$ & $\begin{array}{l}\text { Hong Li, lles } \\
\text { Moraton }\end{array}$ & Picquier & 2008 \\
\hline $\begin{array}{l}\text { Huang Chunming 黄春明 } \\
\text { (1937-) }\end{array}$ & 锣 & Le gong & $\begin{array}{l}\text { Emmanuelle } \\
\text { Péchenart, } \\
\text { Anne Wu } \\
\end{array}$ & Actes Sud & 2001 \\
\hline Huang Fan 黄帆 (1950-) & 慈悲的滋味 & $\begin{array}{l}\text { Le goût amer de la } \\
\text { charité }\end{array}$ & $\begin{array}{l}\text { Pierre Charau, } \\
\text { Mathilde } \\
\text { Chou }\end{array}$ & Flammarion & 2004 \\
\hline $\begin{array}{l}\text { Huang Guojun黄国俊 } \\
(1971-2003)\end{array}$ & 麦克风的试音 & Essais de micro & $\begin{array}{l}\text { Esther Lin, } \\
\text { Angel Pino }\end{array}$ & Actes Sud & 2009 \\
\hline Jia Pingwa 贾平凹 (1952-) & 土门 & Le village englouti & $\begin{array}{l}\text { Genieviève } \\
\text { Imbot-Bichet }\end{array}$ & Stock & 2000 \\
\hline Jiang Rong 姜戎 (1946-) & 狼图腾 & Le totem du loup & $\begin{array}{l}\text { Yan } \\
\text { Hansheng, } \\
\text { Lisa Carducci }\end{array}$ & Bourrin & 2007 \\
\hline Jiang Yun 蒋韵 (1954-) & 现场逃逸 & Délit de fuite & $\begin{array}{l}\text { Myriam } \\
\text { Kryger }\end{array}$ & $\begin{array}{l}\text { Mercure de } \\
\text { France }\end{array}$ & 2001 \\
\hline $\begin{array}{l}\text { Jiang Zidan 蒋子丹 } \\
(1954-)\end{array}$ & 左手 & La main gauche & $\begin{array}{l}\text { Françoise } \\
\text { Naour }\end{array}$ & $\begin{array}{l}\text { Bleu de } \\
\text { Chine }\end{array}$ & 2001 \\
\hline $\begin{array}{l}\text { Jiang Zidan 蒋子丹 } \\
(1954-)\end{array}$ & 桑烟为谁升起 & $\begin{array}{l}\text { Pour qui s'élève la } \\
\text { fumée des mûriers? }\end{array}$ & Prune Cornet & $\begin{array}{l}\text { Bleu de } \\
\text { Chine }\end{array}$ & 2004 \\
\hline Jiao Tong 焦桐 (1956-) & 完全壮阳食谱 & $\begin{array}{l}\text { Recettes aphrodi- } \\
\text { siaques }\end{array}$ & $\begin{array}{l}\text { Marie } \\
\text { Laureillard- } \\
\text { Wendland } \\
\end{array}$ & $\begin{array}{l}\text { Tigre de } \\
\text { papier }\end{array}$ & 2009 \\
\hline $\begin{array}{l}\text { Jidi Majia 吉狄马加 } \\
\text { (1961-) }\end{array}$ & 时间 & Temps & $\begin{array}{l}\text { Sandrine } \\
\text { Alexandre }\end{array}$ & You Feng & 2007 \\
\hline Jin Yong 金庸 (1924-) & 射雕英雄传 & $\begin{array}{l}\text { La légende du héros } \\
\text { chasseur d'aigle }\end{array}$ & $\begin{array}{l}\text { Wang } \\
\text { Jiann-yuh }\end{array}$ & You Feng & 2004 \\
\hline Jin Yong 金庸 (1924-) & 神雕侠侣 & $\begin{array}{l}\text { Le justicier et l'aigle } \\
\text { mythique }\end{array}$ & $\begin{array}{l}\text { Nicole } \\
\text { Tagnon, Xie } \\
\text { Weidong }\end{array}$ & You Feng & 2013 \\
\hline Jiu Dan 九丹 (1968-) & 乌鸦 & Filles-dragons & André Lévy & $\begin{array}{l}\text { Bleu de } \\
\text { Chine }\end{array}$ & 2002 \\
\hline Lao She 老舍 (1899-1966) & 四世同堂 & $\begin{array}{l}\text { Quatre générations } \\
\text { sous le même toit, } \\
\text { tome III (la famine) }\end{array}$ & $\begin{array}{l}\text { Chantal } \\
\text { Chen-Andro }\end{array}$ & $\begin{array}{l}\text { Mercure de } \\
\text { France }\end{array}$ & 2000 \\
\hline Lao She 老舍 (1899-1966) & 二马 & $\begin{array}{l}\text { Messieurs Ma, père et } \\
\text { fils }\end{array}$ & Claude Payen & Picquier & 2000 \\
\hline Lao She 老舍 (1899-1966) & 不说谎的人 & $\begin{array}{l}\text { L'homme qui ne } \\
\text { mentait jamais }\end{array}$ & Claude Payen & Picquier & 2003 \\
\hline Lao She 老舍 (1899-1966) & 老张的哲学 & $\begin{array}{l}\text { La philosophie de } \\
\text { Lao Zhang }\end{array}$ & Claude Payen & Picquier & 2009 \\
\hline Lao She 老舍 (1899-1966) & 文博士 & M.Wen, $\mathrm{PhD}$ & $\begin{array}{l}\text { Bernard } \\
\text { Lelarge }\end{array}$ & You Feng & 2013 \\
\hline
\end{tabular}




\begin{tabular}{|c|c|c|c|c|c|}
\hline Auteur & Titre chinois & Titre français & Traducteur & Éditeur & Parution \\
\hline Lai Niu 老妞 (1966-) & 蹦腾III & Pentium III & $\begin{array}{l}\text { Véronique } \\
\text { Chevaleyre, } \\
\text { Geneviviève } \\
\text { Clastres }\end{array}$ & $\begin{array}{l}\text { Bleu de } \\
\text { Chine }\end{array}$ & 2002 \\
\hline Li Ang 李昂 (1952-) & 迷园 & $\begin{array}{l}\text { Le jardin des } \\
\text { égarements }\end{array}$ & André Levy & Picquier & 2003 \\
\hline Li Ang 李昂 (1952-) & 暗夜 & Nuit obscure & $\begin{array}{l}\text { Marie } \\
\text { Laureillard }\end{array}$ & Actes Sud & 2004 \\
\hline Li Ang 李昂 (1952-) & 杀夫 & Tuer son mari & $\begin{array}{l}\text { Alain } \\
\text { Peyraube, } \\
\text { Hua-Fang } \\
\text { Vizcarra }\end{array}$ & Denoël & 2004 \\
\hline Li Er 李洱 (1966-) & 花腔 & Le jeu du plus fin & Sylvie Gentil & Picquier & 2014 \\
\hline Li Jinxiang 李进祥 (1968-) & $\begin{array}{l}\text { 女人的河（合 } \\
\text { 集） }\end{array}$ & La rivière des femmes & $\begin{array}{l}\text { Françoise } \\
\text { Naour }\end{array}$ & Gallimard & 2012 \\
\hline Li Rui 李锐 (1950-) & 无风之树 & Arbres sans vent & $\begin{array}{l}\text { Annie Curien, } \\
\text { Liu Hongyu }\end{array}$ & Picquier & 2000 \\
\hline Liao Yiwu 廖亦武 (1958-) & 帝国底层 & $\begin{array}{l}\text { Lempire des } \\
\text { bas-fonds }\end{array}$ & $\begin{array}{l}\text { Marie } \\
\text { Holzman } \\
\end{array}$ & $\begin{array}{l}\text { Bleu de } \\
\text { Chine }\end{array}$ & 2003 \\
\hline Liao Yiwu 廖亦武 (1958-) & $\begin{array}{l}\text { 监狱诗: 大屠杀 } \\
\text { - 安眠的灵魂 }\end{array}$ & Poèmes de prison & $\begin{array}{l}\text { Shanshan Sun, } \\
\text { Anne-Marie } \\
\text { Jeanjean }\end{array}$ & L'Harmattan & 2008 \\
\hline Liao Yiwu 廖亦武 (1958-) & 大地震记事 & $\begin{array}{l}\text { Quand la terre } \\
\text { s'ouvre au Sichuan: } \\
\text { journal d'une } \\
\text { tragédie }\end{array}$ & $\begin{array}{l}\text { Marc } \\
\text { Raimbourg }\end{array}$ & $\begin{array}{l}\text { Buchet- } \\
\text { Chastel }\end{array}$ & 2010 \\
\hline Liao Yiwu 廖亦武 (1958-) & 沉沦的圣殿 & $\begin{array}{l}\text { Dans l'empire des } \\
\text { ténèbres }\end{array}$ & $\begin{array}{l}\text { Marc } \\
\text { Raimbourg, } \\
\text { Marie } \\
\text { Holzman } \\
\end{array}$ & $\begin{array}{l}\text { François } \\
\text { Bourin }\end{array}$ & 2013 \\
\hline Liao Zixin 廖子馨 (1964-) & 奥戈的幻觉世界 & $\begin{array}{l}\text { Les hallucinations } \\
\text { d'Ao Ge }\end{array}$ & $\begin{array}{l}\text { Françoise } \\
\text { Naour }\end{array}$ & $\begin{array}{l}\text { Bleu de } \\
\text { Chine }\end{array}$ & 2003 \\
\hline $\begin{array}{l}\text { Liu Qingbang 刘庆邦 } \\
(1951-)\end{array}$ & 神木 & Le puits & $\begin{array}{l}\text { Marianne } \\
\text { Lepolard }\end{array}$ & $\begin{array}{l}\text { Bleu de } \\
\text { Chine }\end{array}$ & 2003 \\
\hline $\begin{array}{l}\text { Liu Qingbang 刘庆邦 } \\
(1951-)\end{array}$ & 灾变 & Cataclysme & $\begin{array}{l}\text { Françoise } \\
\text { Naour }\end{array}$ & Gallimard & 2012 \\
\hline Liu Suola 刘索拉 (1955-) & $\begin{array}{l}\text { 女贞汤: 猪龟娘 } \\
\text { 娘的故事 }\end{array}$ & $\begin{array}{l}\text { La grande île des } \\
\text { tortues-cochons }\end{array}$ & Sylvie Gentil & Seuil & 2006 \\
\hline Liu Xiaobo 刘晓波 (1955-) & 猪的哲学 & $\begin{array}{l}\text { La philosophie du } \\
\text { porc, et autres essais }\end{array}$ & $\begin{array}{l}\text { Jea-Philippe } \\
\text { Béja }\end{array}$ & $\begin{array}{l}\text { Gallimard, } \\
\text { Bleu de } \\
\text { Chine }\end{array}$ & 2011 \\
\hline Liu Xiaobo 刘晓波 (1955-) & $?$ & Vivre dans la vérité & $\begin{array}{l}\text { Geneviève } \\
\text { Imbot-Bichet }\end{array}$ & $\begin{array}{l}\text { Gallimard, } \\
\text { Bleu de } \\
\text { Chine }\end{array}$ & 2012 \\
\hline Liu Xinwu 刘心武 (1942-) & 如意 & Le talisman & R.Y.L. Yo & You Feng & 2000 \\
\hline Liu Xinwu 刘心武 (1942-) & $\begin{array}{l}\text { 护城河边的灰姑 } \\
\text { 娘 }\end{array}$ & $\begin{array}{l}\text { La Cendrillon du } \\
\text { canal }\end{array}$ & $\begin{array}{l}\text { Roger } \\
\text { Darrobers }\end{array}$ & $\begin{array}{l}\text { Bleu de } \\
\text { Chine }\end{array}$ & 2003 \\
\hline Liu Xinwu 刘心武 (1942-) & 树与林同在 & $\begin{array}{l}\text { L'arbre et la forêt: } \\
\text { destins croisés }\end{array}$ & $\begin{array}{l}\text { Roger } \\
\text { Darrobers }\end{array}$ & $\begin{array}{l}\text { Bleu de } \\
\text { Chine }\end{array}$ & 2003 \\
\hline Liu Xinwu 刘心武 (1942-) & 老舍之死 & La mort de Lao She & $\begin{array}{l}\text { Françoise } \\
\text { Naour }\end{array}$ & $\begin{array}{l}\text { Bleu de } \\
\text { Chine }\end{array}$ & 2004 \\
\hline Liu Xinwu 刘心武 (1942-) & 民工老何 & Poussière et sueur & $\begin{array}{l}\text { Roger } \\
\text { Darrobers }\end{array}$ & Gallimard & 2004 \\
\hline Liu Xinwu 刘心武 (1942-) & 人面鱼 & $\begin{array}{l}\text { Poisson à face } \\
\text { humaine }\end{array}$ & $\begin{array}{l}\text { Roger } \\
\text { Darrobers }\end{array}$ & $\begin{array}{l}\text { Bleu de } \\
\text { Chine }\end{array}$ & 2004 \\
\hline Liu Xinwu 刘心武 (1942-) & 蓝夜叉 & La démone bleue & $\begin{array}{l}\text { Roger } \\
\text { Darrobers }\end{array}$ & $\begin{array}{l}\text { Bleu de } \\
\text { Chine }\end{array}$ & 2005 \\
\hline
\end{tabular}




\begin{tabular}{|c|c|c|c|c|c|}
\hline Auteur & Titre chinois & Titre français & Traducteur & Éditeur & Parution \\
\hline Liu Xinwu 刘心武 (1942-) & 泼妇鸡丁 & $\begin{array}{l}\text { Dés de poulet façon } \\
\text { mégère }\end{array}$ & $\begin{array}{l}\text { Marie } \\
\text { Laureillard }\end{array}$ & $\begin{array}{l}\text { Bleu de } \\
\text { Chine }\end{array}$ & 2007 \\
\hline Liu Xinwu 刘心武 (1942-) & $\begin{array}{l}\text { 生于6月4日-刘心 } \\
\text { 武回忆录 }\end{array}$ & $\begin{array}{l}\text { Je suis né un } 4 \text { juin. } \\
\text { Mémoires littéraires }\end{array}$ & $\begin{array}{l}\text { Marie } \\
\text { Laureillard }\end{array}$ & $\begin{array}{l}\text { Gallimard, } \\
\text { Bleu de } \\
\text { Chine }\end{array}$ & 2013 \\
\hline Liu Xinwu 刘心武 (1942-) & 冒牌城市 & $\begin{array}{l}\text { La guérite, la force } \\
\text { des farces en terre } \\
\text { chinoise }\end{array}$ & $\begin{array}{l}\text { Françoise } \\
\text { Naour }\end{array}$ & $\begin{array}{l}\text { Bleu de } \\
\text { Chine }\end{array}$ & 2006 \\
\hline Liu Xinwu 刘心武 (1942-) & 挑担茶叶上北京 & $\begin{array}{l}\text { Du thé d'hiver pour } \\
\text { Pékin }\end{array}$ & $\begin{array}{l}\text { Françoise } \\
\text { Naour }\end{array}$ & $\begin{array}{l}\text { Bleu de } \\
\text { Chine }\end{array}$ & 2004 \\
\hline $\begin{array}{l}\text { Liu Yichang 刘以榰 } \\
\text { (1918-) }\end{array}$ & 对倒 & Tête-bêche & $\begin{array}{l}\text { Pascale } \\
\text { Wei-Guinot }\end{array}$ & Picquier & 2003 \\
\hline $\begin{array}{l}\text { Liu Zhenyun刘震云 } \\
\text { (1958-) }\end{array}$ & 官人 & Les Mandarins & Sebastian Veg & $\begin{array}{l}\text { Bleu de } \\
\text { Chine }\end{array}$ & 2003 \\
\hline $\begin{array}{l}\text { Liu Zhenyun 刘震云 } \\
(1958-)\end{array}$ & 一地鸡毛 & $\begin{array}{l}\text { Peaux d'ail et plumes } \\
\text { de poulet }\end{array}$ & Sebastian Veg & $\begin{array}{l}\text { Bleu de } \\
\text { Chine }\end{array}$ & 2006 \\
\hline $\begin{array}{l}\text { Liu Zhenyun刘震云 } \\
(1958-)\end{array}$ & 温故1942 & Se souvenir de 1942 & $\begin{array}{l}\text { Geneviève } \\
\text { Imbot-Bichet }\end{array}$ & $\begin{array}{l}\text { Gallimard, } \\
\text { Bleu de } \\
\text { Chine }\end{array}$ & 2013 \\
\hline $\begin{array}{l}\text { Liu Zhenyun 刘震云 } \\
\text { (1958-) }\end{array}$ & 一句顶一万句 & $\begin{array}{l}\text { En un mot comme } \\
\text { en mille }\end{array}$ & $\begin{array}{l}\text { Isabelle Bijon, } \\
\text { Wang } \\
\text { Jiann-Yuh }\end{array}$ & $\begin{array}{l}\text { Gallimard, } \\
\text { Bleu de } \\
\text { Chine }\end{array}$ & 2013 \\
\hline $\begin{array}{l}\text { Lu Wenfu 陆文夫 } \\
\text { (1928-2005) }\end{array}$ & 人之窝 & Nid d'hommes & $\begin{array}{l}\text { Chantal } \\
\text { Chen-Andro }\end{array}$ & Seuil & 2004 \\
\hline Lu Xun 鲁迅 (1881-1936) & 彷徨 & Errances & $\begin{array}{l}\text { Jacques } \\
\text { Meunier }\end{array}$ & You Feng & 2004 \\
\hline Lu Xun 鲁迅 (1881-1936) & 彷得 & Errances & Sebastian Veg & Rue d'Ulm & 2004 \\
\hline Lu Xun 鲁迅 (1881-1936) & 呐喊 & Cris & Sebastian Veg & Rue d'Ulm & 2010 \\
\hline Luo Ying 骆英 (1956-) & 小兔子 & Lapins, lapins & Shuang Xu & Castor astral & 2013 \\
\hline $\begin{array}{l}\text { Lü Tiancheng 吕天成 } \\
(1580-1618)\end{array}$ & 绣榻野史 & $\begin{array}{l}\text { Histoire hétérodoxe } \\
\text { d'un lit brodé }\end{array}$ & $\begin{array}{l}\text { Huang San et } \\
\text { Lionel Epstein }\end{array}$ & Picquier & 2001 \\
\hline Lü You’an 吕幼安 (1950-) & 我没有错 & Ce n'est pas ma faute & $\begin{array}{l}\text { Philippe } \\
\text { Denizet }\end{array}$ & You Feng & 2003 \\
\hline $\begin{array}{l}\text { Ma Desheng 马德升 } \\
(1952-)\end{array}$ & 白梦, 黑魂 & $\begin{array}{l}\text { Rêve blanc, âmes } \\
\text { noires }\end{array}$ & $\begin{array}{l}\text { Emmanuelle } \\
\text { Péchenart }\end{array}$ & L'Aube & 2003 \\
\hline $\begin{array}{l}\text { Ma Xiaoquan 马笑泉 } \\
(1978-)\end{array}$ & 愤怒青年 & $\begin{array}{l}\text { Confession d'un } \\
\text { tueur à gages }\end{array}$ & $\begin{array}{l}\text { Marie-Claude } \\
\text { Cantournet- } \\
\text { Jacquet }\end{array}$ & L'Olivier & 2006 \\
\hline $\begin{array}{l}\text { Mao Dun 茅盾 (1896- } \\
\text { 1981) }\end{array}$ & 子夜 & Minuit & $\begin{array}{l}\text { Jacques } \\
\text { Meunier, } \\
\text { Michelle Loi }\end{array}$ & You Feng & 2011 \\
\hline Meng Ming 孟明 (1955-) & 槐花之年 & $\begin{array}{l}\text { L'année des fleurs de } \\
\text { sophora }\end{array}$ & $\begin{array}{l}\text { Emmanuelle } \\
\text { Péchenart }\end{array}$ & Cheyne & 2011 \\
\hline Main Mian 棉棉 (1970-) & 糖 & Les bonbons chinois & Sylvie Gentil & L'Olivier & 2001 \\
\hline- & 熊猫 & Panda sex & - & $\begin{array}{l}\text { Au diable } \\
\text { Vauvert }\end{array}$ & 2009 \\
\hline Mo Ren 墨人 (1920-) & 红尘 & Poussière rouge & Wang Chia-yu & You-Feng & 2004 \\
\hline Mo Yan 莫言 (1955-) & 酒国 & Le pays de l'alcool & $\begin{array}{l}\text { Liliane et Noël } \\
\text { Dutrait }\end{array}$ & Seuil & 2000 \\
\hline Mo Yan 莫言 (1955-) & 丰乳肥臀 & $\begin{array}{l}\text { Beaux seins, belles } \\
\text { fesses: les enfants de } \\
\text { la famille Shangguan }\end{array}$ & $\begin{array}{l}\text { Noël et Liliane } \\
\text { Dutrait }\end{array}$ & Seuil & 2004 \\
\hline Mo Yan 莫言 (1955-) & 藏宝图 & La carte au trésor & $\begin{array}{l}\text { Antoine } \\
\text { Ferragne }\end{array}$ & Picquier & 2004 \\
\hline Mo Yan 莫言 (1955-) & 铁孩子 & Enfant de fer & $\begin{array}{l}\text { Chantal } \\
\text { Chen-Andro }\end{array}$ & Seuil & 2004 \\
\hline
\end{tabular}




\begin{tabular}{|c|c|c|c|c|c|}
\hline Auteur & Titre chinois & Titre français & Traducteur & Éditeur & Parution \\
\hline Mo Yan 莫言 (1955-) & 师傅越来越幽默 & $\begin{array}{l}\text { Le maitre a de plus } \\
\text { en plus d'humour }\end{array}$ & Noël Dutrait & Seuil & 2005 \\
\hline Mo Yan 莫言 (1955-) & 檀香刑 & Le supplice du santal & $\begin{array}{l}\text { Chantal } \\
\text { Chen-Andro }\end{array}$ & Seuil & 2006 \\
\hline Mo Yan 莫言 (1955-) & 欢乐 & La joie & $\begin{array}{l}\text { Marie } \\
\text { Laureillard }\end{array}$ & Picquier & 2007 \\
\hline Mo Yan 莫言 (1955-) & 四十一炮 & $\begin{array}{l}\text { Quarante et un coups } \\
\text { de canon }\end{array}$ & $\begin{array}{l}\text { Noël et Liliane } \\
\text { Dutrait }\end{array}$ & Picquier & 2008 \\
\hline Mo Yan 莫言 (1955-) & 生死疲劳 & La dure loi du karma & $\begin{array}{l}\text { Chantal } \\
\text { Chen-Andro }\end{array}$ & Seuil & 2009 \\
\hline Mo Yan 莫言 (1955-) & $\begin{array}{l}\text { 长安大道上的骑 } \\
\text { 驴美人 }\end{array}$ & $\begin{array}{l}\text { La belle à dos d'âne } \\
\text { dans l'avenue de } \\
\text { Chang'an }\end{array}$ & $\begin{array}{l}\text { Marie } \\
\text { Laureillard }\end{array}$ & Picquier & 2011 \\
\hline Mo Yan 莫言 (1955-) & 蛙 & Grenouilles & $\begin{array}{l}\text { Chantal } \\
\text { Chen-Andro }\end{array}$ & Seuil & 2011 \\
\hline Mo Yan 莫言 (1955-) & 牛 & Le veau & $\begin{array}{l}\text { François } \\
\text { Sastourné }\end{array}$ & Seuil & 2012 \\
\hline Mo Yan 莫言 (1955-) & 变 & Le grand chambard & $\begin{array}{l}\text { Chantal } \\
\text { Chen-Andro }\end{array}$ & Seuil & 2013 \\
\hline Mo Yan 莫言 (1955-) & 五爱街 & $\begin{array}{l}\text { Le marché des } \\
\text { amours et des peines }\end{array}$ & $\begin{array}{l}\text { Rebecca } \\
\text { Peyrelon }\end{array}$ & Seuil & 2004 \\
\hline $\begin{array}{l}\text { Murong Xuecun 慕容雪村 } \\
(1974-)\end{array}$ & $\begin{array}{l}\text { 成都, 今夜请将 } \\
\text { 我遗忘 }\end{array}$ & Oublier Chengdu & Claude Payen & L'Olivier & 2006 \\
\hline 慕容雪村 (1974-) & 原谅我红尘颠倒 & $\begin{array}{l}\text { Danse dans la } \\
\text { poussière rouge }\end{array}$ & Claude Payen & Gallimard & 2013 \\
\hline $\begin{array}{l}\text { Qiu Huadong 邱华栋 } \\
\text { (1969-) }\end{array}$ & 遗忘者之路 & $\begin{array}{l}\text { Voyage au pays de } \\
\text { loubli }\end{array}$ & Claire Yang & $\begin{array}{l}\text { Bleu de } \\
\text { Chine }\end{array}$ & 2001 \\
\hline $\begin{array}{l}\text { Qiu Huadong 邱华栋 } \\
\text { (1969-) }\end{array}$ & $\begin{array}{l}\text { 黑暗河流上的闪 } \\
\text { 光 }\end{array}$ & $\begin{array}{l}\text { Reflets sur la rivière } \\
\text { obscure }\end{array}$ & Claire Yang & $\begin{array}{l}\text { Bleu de } \\
\text { Chine }\end{array}$ & 2002 \\
\hline $\begin{array}{l}\text { Qiu Huadong 邱华栋 } \\
\text { (1969-) }\end{array}$ & 手上的星光 & $\begin{array}{l}\text { Scintillement sur la } \\
\text { main }\end{array}$ & $\begin{array}{l}\text { Eric } \\
\text { Jacquemin }\end{array}$ & $\begin{array}{l}\text { Bleu de } \\
\text { Chine }\end{array}$ & 2004 \\
\hline Shang Qi商禽 (1930-) & 梦或者黎明 & $\begin{array}{l}\text { Rêve ou aube: } \\
\text { poèmes }\end{array}$ & $\begin{array}{l}\text { Martine } \\
\text { Vallette- } \\
\text { Hémery }\end{array}$ & Murmure & 2005 \\
\hline $\begin{array}{l}\text { Shen Congwen沈从文 } \\
(1902-1988)\end{array}$ & 湘行散记 & $\begin{array}{l}\text { Le périple de Xiang } \\
\text { et autres nouvelles }\end{array}$ & $\begin{array}{l}\text { Gilles } \\
\text { Cabrero, } \\
\text { Marie } \\
\text { Laureillard }\end{array}$ & Gallimard & 2012 \\
\hline Shen Shixi 沈石溪 (1952-) & 狼王梦 & Le rêve d'Alpha & $\begin{array}{l}\text { Mathilde } \\
\text { Colo-Wu }\end{array}$ & $\begin{array}{l}\text { Horizon } \\
\text { oriental \& } \\
\text { Cèdre Lune }\end{array}$ & 2013 \\
\hline $\begin{array}{l}\text { Shi Shuqing 施叔青 } \\
\text { (1945-) }\end{array}$ & 她名叫蝴蝶 & Elle s'appelle Papillon & $\begin{array}{l}\text { Jiann-Yuh } \\
\text { Wang }\end{array}$ & Herne & 2004 \\
\hline $\begin{array}{l}\text { Shi Tiesheng 史铁生 } \\
\text { (1951-2010) }\end{array}$ & 宿命 & Fatalité & Annie Curien & Gallimard & 2004 \\
\hline $\begin{array}{l}\text { Shi Zhecun 施蛰存 } \\
(1905-2003)\end{array}$ & 雨的滋味 & $\begin{array}{l}\text { Le goût de la pluie: } \\
\text { nouvelles et prose de } \\
\text { circonstance }\end{array}$ & $\begin{array}{l}\text { Gilles } \\
\text { Cabrero, } \\
\text { Marie } \\
\text { Laureillard }\end{array}$ & Gallimard & 2011 \\
\hline Song Lin 宋琳 (1959-) & 城墙与落日 & $\begin{array}{l}\text { Murailles et } \\
\text { couchants }\end{array}$ & $\begin{array}{l}\text { Chantal } \\
\text { Chen-Andro }\end{array}$ & Caractères & 2007 \\
\hline Su Tong 苏童 (1963-) & 我的帝王生涯 & $\begin{array}{l}\text { Je suis l'empereur de } \\
\text { Chine }\end{array}$ & Claude Payen & Picquier & 2005 \\
\hline Su Tong 苏童 (1963-) & 碧奴 & Le mythe de Meng & $\begin{array}{l}\text { Marie } \\
\text { Laureillard }\end{array}$ & Flammarion & 2009 \\
\hline Su Tong 苏童 (1963-) & 自行车之歌 & À bicyclette & $\begin{array}{l}\text { Anne-Laure } \\
\text { Fournier }\end{array}$ & Picquier & 2011 \\
\hline
\end{tabular}




\begin{tabular}{|c|c|c|c|c|c|}
\hline Auteur & Titre chinois & Titre français & Traducteur & Éditeur & Parution \\
\hline Su Tong 苏童 (1963-) & 河岸 & La berge & $\begin{array}{l}\text { François } \\
\text { Sastourné }\end{array}$ & Gallimard & 2011 \\
\hline Tian Yuan 田原 (1985-) & 斑马森林 & La forêt zèbre & Sylvie Gentil & L'Olivier & 2002 \\
\hline Tie Ning 铁凝 (1957-) & 第十二夜 & La douzième nuit & Prune Cornet & $\begin{array}{l}\text { Bleu de } \\
\text { Chine }\end{array}$ & 2004 \\
\hline Tie Ning 铁凝 (1957-) & 笨花 & Fleurs de coton & $\begin{array}{l}\text { Véronique } \\
\text { Chevaleyre }\end{array}$ & $\begin{array}{l}\text { Bleu de } \\
\text { Chine }\end{array}$ & 2005 \\
\hline Wang Anyi 王安忆 (1954-) & 香港的情与爱 & $\begin{array}{l}\text { Les lumières de Hong } \\
\text { Kong }\end{array}$ & $\begin{array}{l}\text { Denis } \\
\text { Bénéjam }\end{array}$ & Picquier & 2001 \\
\hline Wang Anyi 王安忆 (1954-) & 长恨歌 & $\begin{array}{l}\text { Le chant des regrets } \\
\text { éternels }\end{array}$ & $\begin{array}{l}\text { Yvonne } \\
\text { André, } \\
\text { Stéphane } \\
\text { Lévêque }\end{array}$ & Picquier & 2006 \\
\hline Wang Anyi 王安忆 (1954-) & 小城之恋 & $\begin{array}{l}\text { Amour dans une } \\
\text { petite ville }\end{array}$ & Yvonne André & Picquier & 2007 \\
\hline Wang Anyi 王安忆 (1954-) & 绵乡谷之恋 & $\begin{array}{l}\text { Amour dans une } \\
\text { vallée enchantée }\end{array}$ & Yvonne André & Picquier & 2008 \\
\hline Wang Anyi 王安忆 (1954-) & 荒山之恋 & $\begin{array}{l}\text { Amour sur une } \\
\text { colline dénudée }\end{array}$ & $\begin{array}{l}\text { Stéphane } \\
\text { Lévêque }\end{array}$ & Picquier & 2008 \\
\hline Wang Anyi 王安忆 (1954-) & 寻找上海 & $\begin{array}{l}\text { À la recherche de } \\
\text { Shanghai }\end{array}$ & Yvonne André & Picquier & 2013 \\
\hline Wang Anyi 安忆 (1954-) & 月色撩人 & $\begin{array}{l}\text { Le plus clair de la } \\
\text { lune }\end{array}$ & Yvonne André & Picquier & 2013 \\
\hline Wang Chao 王超 (1964-) & 安阳孤儿 & L'orphelin d'Anyang & $\begin{array}{l}\text { Cecile } \\
\text { Delattre }\end{array}$ & $\begin{array}{l}\text { Bleu de } \\
\text { Chine }\end{array}$ & 2002 \\
\hline Wang Chao 王超 (1964-) & 去了西藏 & Tibet sans retour & $\begin{array}{l}\text { Françoise } \\
\text { Naour }\end{array}$ & $\begin{array}{l}\text { Bleu de } \\
\text { Chine }\end{array}$ & 2002 \\
\hline Wang Chao 王超 (1964-) & 南方 & $\begin{array}{l}\text { Homme du Sud, } \\
\text { femme du Nord }\end{array}$ & $\begin{array}{l}\text { Françoise } \\
\text { Naour }\end{array}$ & Page à page & 2004 \\
\hline Wang Chao 王超 (1964-) & 天堂有爱 & Au paradis, l'amour & $\begin{array}{l}\text { Cecile } \\
\text { Delattre, } \\
\text { Jean-Marie } \\
\text { Casanova }\end{array}$ & $\begin{array}{l}\text { Bleu de } \\
\text { Chine }\end{array}$ & 2004 \\
\hline $\begin{array}{l}\text { Wang Dulu 王度庐 } \\
(1909-1977)\end{array}$ & 卧龙藏虎 & Tigre et dragon & $\begin{array}{l}\text { Solange } \\
\text { Cruveillé, } \\
\text { Amélie } \\
\text { Manon }\end{array}$ & $\begin{array}{l}\text { Calmann- } \\
\text { Lévy }\end{array}$ & 2007 \\
\hline Wang Gang 王刚 (1960-) & 英格力士 & English & $\begin{array}{l}\text { Pascale } \\
\text { Wei-Guinot, } \\
\text { Emmanuelle } \\
\text { Péchenart }\end{array}$ & Picquier & 2008 \\
\hline $\begin{array}{l}\text { Wang Jiaxiang王嘉祥 } \\
(1966-)\end{array}$ & 小矮人之谜 & $\begin{array}{l}\text { À la recherche des } \\
\text { petits hommes }\end{array}$ & Elsa Chalaux & $\begin{array}{l}\text { Tigre de } \\
\text { papier }\end{array}$ & 2009 \\
\hline Wang Meng 王蒙 (1934-) & 淡灰色的眼珠 & Des yeux gris clair & $\begin{array}{l}\text { Françoise } \\
\text { Naour }\end{array}$ & $\begin{array}{l}\text { Bleu de } \\
\text { Chine }\end{array}$ & 2002 \\
\hline Wang Meng 王蒙 (1934-) & 笑而不答 & Les sourires du sage & $\begin{array}{l}\text { Françoise } \\
\text { Naour }\end{array}$ & $\begin{array}{l}\text { Bleu de } \\
\text { Chine }\end{array}$ & 2003 \\
\hline Wang Meng 王蒙 (1934-) & 跳舞 & Celle qui dansait & $\begin{array}{l}\text { Françoise } \\
\text { Naour }\end{array}$ & $\begin{array}{l}\text { Bleu de } \\
\text { Chine }\end{array}$ & 2004 \\
\hline $\begin{array}{l}\text { Wang Xiaobo 王小波 } \\
(1952-1997)\end{array}$ & 黄金时代 & Lâge d'or & Jacques Seurre & Sorgho & 2001 \\
\hline $\begin{array}{l}\text { Wang Xiaobo 王小波 } \\
\text { (1952-1997) }\end{array}$ & 王小波散文选 & $\begin{array}{l}\text { La majorité } \\
\text { silencieuse }\end{array}$ & $\begin{array}{l}\text { Luc } \\
\text { Thominette, } \\
\text { Bai Yunfei }\end{array}$ & You Feng & 2013 \\
\hline $\begin{array}{l}\text { Wang Xiaobo 王小波 } \\
\text { (1952-1997) }\end{array}$ & 未来世界 & Le monde futur & Mei Mercier & Actes Sud & 2013 \\
\hline
\end{tabular}




\begin{tabular}{|c|c|c|c|c|c|}
\hline Auteur & Titre chinois & Titre français & Traducteur & Éditeur & Parution \\
\hline Wang Xingyu (1977-) & 杨浦小流讯 & $\begin{array}{l}\text { Petit voyou de } \\
\text { l'arrondissement de } \\
\text { Yang Pu }\end{array}$ & $\begin{array}{l}\text { Jean Testard, } \\
\text { Olivier } \\
\text { Descour }\end{array}$ & L'Harmattan & 2005 \\
\hline $\begin{array}{l}\text { Wu Mingyi 吴明益 } \\
\text { (1971-) }\end{array}$ & 睡眠的航线 & $\begin{array}{l}\text { Les lignes de } \\
\text { navigation du } \\
\text { sommeil }\end{array}$ & $\begin{array}{l}\text { Gwennaël } \\
\text { Gaffric }\end{array}$ & You Feng & 2013 \\
\hline Xi Yang 西岉 (1965-) & 青衣花旦 & $\begin{array}{l}\text { La rêveuse et la } \\
\text { dragueuse }\end{array}$ & $\begin{array}{l}\text { Françoise } \\
\text { Naour }\end{array}$ & $\begin{array}{l}\text { Bleu de } \\
\text { Chine }\end{array}$ & 2002 \\
\hline Xia Yan 夏衍 (1900-1995) & 上海屋檐下 & $\begin{array}{l}\text { Sous les toits de } \\
\text { Shanghai }\end{array}$ & $\begin{array}{l}\text { Rébecca } \\
\text { Peyrelon }\end{array}$ & You Feng & 2001 \\
\hline Xia Yan 夏衍 (1900-1995) & 法西斯细菌 & Le virus du fascisme & $\begin{array}{l}\text { Rébecca } \\
\text { Peyrelon }\end{array}$ & You Feng & 2002 \\
\hline $\begin{array}{l}\text { Xiaman Lanbo'an 夏曼·蓝 } \\
\text { 波安 (1957-) }\end{array}$ & 波浪的记忆 & $\begin{array}{l}\text { La mémoire des } \\
\text { vagues }\end{array}$ & $\begin{array}{l}\text { Marie-Paule } \\
\text { Chamayou }\end{array}$ & $\begin{array}{l}\text { Tigre de } \\
\text { papier }\end{array}$ & 2011 \\
\hline $\begin{array}{l}\text { Xiao Hong 萧红 } \\
(1911-1942)\end{array}$ & 呼兰河传 & $\begin{array}{l}\text { Contes de la rivière } \\
\text { Hulan }\end{array}$ & $\begin{array}{l}\text { Simone } \\
\text { Cros-Moréa }\end{array}$ & $\begin{array}{l}\text { Tigre de } \\
\text { papier }\end{array}$ & 2011 \\
\hline Xin Ran 欣然 (1958-) & 筷子姑娘 & Baguettes chinoises & Prune Cornet & Picquier & 2008 \\
\hline Xin Ran 欣然 (1958-) & $\begin{array}{l}\text { 中国民族自尊的 } \\
\text { 见证者们 }\end{array}$ & $\begin{array}{l}\text { Mémoire de Chine: } \\
\text { les voix d'une } \\
\text { génération } \\
\text { silencieuse }\end{array}$ & Prune Cornet & Picquier & 2010 \\
\hline Xu Shuya 徐舒雅 (1961-) & 八月雪 & $\begin{array}{l}\text { La neige en août: } \\
\text { épopée lyrique en } \\
\text { deux actes }\end{array}$ & $\begin{array}{l}\text { André Segond, } \\
\text { Noel et Liliane } \\
\text { Dutrait }\end{array}$ & Actes Sud & 2004 \\
\hline Xu Xing 徐星 (1956-) & 剩下的都属于你 & $\begin{array}{l}\text { Et tout ce qui reste } \\
\text { est pour toi }\end{array}$ & Sylvie Gentil & L'Olivier & 2003 \\
\hline- & 无主题变奏 & $\begin{array}{l}\text { Variations sans } \\
\text { thème }\end{array}$ & Sylvie Gentil & L'Olivier & 2003 \\
\hline Yan Lianke 阎连科 (1958-) & 为人民服务 & Servir le peuple & Claude Payen & Picquier & 2006 \\
\hline Yan Lianke 阎连科 (1958-) & 丁庄梦 & $\begin{array}{l}\text { Le rêve du village des } \\
\text { Ding }\end{array}$ & Claude Payen & Picquier & 2007 \\
\hline Yan Lianke 阎连科 (1958-) & 年月日 & $\begin{array}{l}\text { Les jours, les mois, les } \\
\text { années }\end{array}$ & $\begin{array}{l}\text { Brigitte } \\
\text { Guilbaud }\end{array}$ & Picquier & 2009 \\
\hline Yan Lianke 阎连科 (1958-) & 受活 & $\begin{array}{l}\text { Bons baisers de } \\
\text { Lénine }\end{array}$ & Sylvie Gentil & Picquier & 2009 \\
\hline Yan Lianke 阎连科 (1958-) & 我与父辈 & Songeant à mon père & $\begin{array}{l}\text { Brigitte } \\
\text { Guilbaud }\end{array}$ & Picquier & 2010 \\
\hline Yan Lianke 阎连科 (1958-) & 四书 & Les quatre livres & Sylvie Gentil & Picquier & 2012 \\
\hline Yan Lianke 阎连科 (1958-) & 日光流年 & La fuite du temps & $\begin{array}{l}\text { Brigitte } \\
\text { Guilbaud }\end{array}$ & Picquier & 2014 \\
\hline Yang Lian 杨炼 (1955-) & 河口上的房间 & $\begin{array}{l}\text { La maison sur } \\
\text { l'estuaire }\end{array}$ & $\begin{array}{l}\text { Chantal } \\
\text { Chen-Andro }\end{array}$ & MEET & 2001 \\
\hline Yang Lian 杨炼 (1955-) & 面具与鳄鱼 & $\begin{array}{l}\text { Masques et } \\
\text { crocodiles }\end{array}$ & $\begin{array}{l}\text { Chantal } \\
\text { Chen-Andro }\end{array}$ & Virgile & 2002 \\
\hline Yang Lian 杨炼 (1955-) & 大海停止之处 & Là où s'arrête la mer & $\begin{array}{l}\text { Chantal } \\
\text { Chen-Andro }\end{array}$ & Carctères & 2004 \\
\hline Yang Lian 杨炼 (1955-) & 幸福鬼魂手记 & $\begin{array}{l}\text { Notes manuscrites } \\
\text { d'un diable heureux }\end{array}$ & $\begin{array}{l}\text { Chantal } \\
\text { Chen-Andro }\end{array}$ & Carctères & 2010 \\
\hline $\begin{array}{l}\text { Yang Zhengguang 杨争光 } \\
\text { (1957-) }\end{array}$ & 老旦是一棵树 & Mon cher ennemi & $\begin{array}{l}\text { Raymond } \\
\text { Rocher, Chen } \\
\text { Xiangrong }\end{array}$ & $\begin{array}{l}\text { Bleu de } \\
\text { Chine }\end{array}$ & 2007 \\
\hline Ye Si 也斯 (1948-) & 岛与大陆 & $\begin{array}{l}\text { Iles et continents, et } \\
\text { autres nouvelles }\end{array}$ & Annie Curien & Gallimard & 2001 \\
\hline Ye Mang 野莽 (1953-) & 打你五十大板 & Intelligence & Lü Hua & $\begin{array}{l}\text { Bleu de } \\
\text { Chine }\end{array}$ & 2003 \\
\hline Ye Mang 野莽 (1953-) & $\begin{array}{l}\text { 玩阿基米德飞盘 } \\
\text { 的王永乐师傅 }\end{array}$ & $\begin{array}{l}\text { Les secrets d'un petit } \\
\text { monde }\end{array}$ & Lü Hua & $\begin{array}{l}\text { Bleu de } \\
\text { Chine }\end{array}$ & 2004 \\
\hline
\end{tabular}




\begin{tabular}{|c|c|c|c|c|c|}
\hline Auteur & Titre chinois & Titre français & Traducteur & Éditeur & Parution \\
\hline $\begin{array}{l}\text { Ye Zhaoyan 叶兆言 } \\
\text { (1957-) }\end{array}$ & 没有玻璃的花房 & La serre sans verre & $\begin{array}{l}\text { Jiann-Yuh } \\
\text { Wang }\end{array}$ & $\begin{array}{l}\text { Bleu de } \\
\text { Chine }\end{array}$ & 2006 \\
\hline $\begin{array}{l}\text { Ye Zhaoyan 叶兆言 } \\
\text { (1957-) }\end{array}$ & 一情 & $\begin{array}{l}\text { Nankin 1937, une } \\
\text { histoire d'amour }\end{array}$ & $\begin{array}{l}\text { Nathalie } \\
\text { Louisgrand- } \\
\text { Thomas }\end{array}$ & Seuil & 2008 \\
\hline Yu Hua 余华 (1960-) & 古典爱情 & Un amour classique & $\begin{array}{l}\text { Jacqueline } \\
\text { Guyvallet }\end{array}$ & Actes Sud & 2000 \\
\hline Yu Hua 余华 (1960-) & 在细雨中呼喊 & Cris dans la bruine & $\begin{array}{l}\text { Jacqueline } \\
\text { Guyvallet }\end{array}$ & Actes Sud & 2003 \\
\hline Yu Hua 余华 (1960-) & 兄弟 & Brothers & $\begin{array}{l}\text { Angel Pino, } \\
\text { Isabelle Rabut }\end{array}$ & Actes Sud & 2008 \\
\hline Yu Hua 余华 (1960-) & 十八岁出门远行 & $\begin{array}{l}\text { Sur la route à } \\
\text { dix-huit ans, et autres } \\
\text { nouvelles }\end{array}$ & $\begin{array}{l}\text { Jacqueline } \\
\text { Guyvallet }\end{array}$ & Actes Sud & 2009 \\
\hline Yu Hua 余华 (1960-) & $\begin{array}{l}\text { 十个词汇里的中 } \\
\text { 国 }\end{array}$ & La Chine en dix mots & $\begin{array}{l}\text { Angel Pino, } \\
\text { Isabelle Rabut }\end{array}$ & Actes Sud & 2010 \\
\hline $\begin{array}{l}\text { Yu Dafu郁达夫 (1896- } \\
\text { 1945) }\end{array}$ & 秋河 & $\begin{array}{l}\text { Rivière d'automne, et } \\
\text { autres nouvelles }\end{array}$ & $\begin{array}{l}\text { Stéphane } \\
\text { Lévêque }\end{array}$ & Picquier & 2002 \\
\hline Yu Jian 于坚 (1954-) & 飞行 & Un vol & $\begin{array}{l}\text { Li Jinjia, } \\
\text { Sébastian Veg }\end{array}$ & $\begin{array}{l}\text { Gallimard, } \\
\text { Bleu de } \\
\text { Chine }\end{array}$ & 2010 \\
\hline $\begin{array}{l}\text { Zhang Ailing 张爱玲 } \\
(1920-1995)\end{array}$ & 红玫瑰与白玫瑰 & $\begin{array}{l}\text { Rose rouge et rose } \\
\text { blanche }\end{array}$ & $\begin{array}{l}\text { Emmanuelle } \\
\text { Péchenart }\end{array}$ & $\begin{array}{l}\text { Bleu de } \\
\text { Chine }\end{array}$ & 2001 \\
\hline $\begin{array}{l}\text { Zhang Ailing 张爱玲 } \\
(1920-1995)\end{array}$ & 倾城之恋 & $\begin{array}{l}\text { Un amour dévasta- } \\
\text { teur }\end{array}$ & $\begin{array}{l}\text { Emmanuelle } \\
\text { Péchenart }\end{array}$ & L'Aube & 2005 \\
\hline $\begin{array}{l}\text { Zhang Ailing 张爱玲 } \\
(1920-1995)\end{array}$ & 色戒 & $\begin{array}{l}\text { Lust, caution (amour, } \\
\text { luxure, trahison) }\end{array}$ & $\begin{array}{l}\text { Emmanuelle } \\
\text { Péchenart }\end{array}$ & Laffont & 2008 \\
\hline Zhang Ling 张翎 (1957-) & 金山 & $\begin{array}{l}\text { Le rêve de la } \\
\text { montagne d'or }\end{array}$ & Claude Payen & Belford & 2012 \\
\hline Zhang Yu 张宇 (1952-...) & 软弱 & Ripoux à Zhengzhou & Claude Payen & Picquier & 2002 \\
\hline $\begin{array}{l}\text { Zhang Yihe 章诒和 } \\
\text { (1942-) }\end{array}$ & 刘氏女 & Madame Liu & $\begin{array}{l}\text { François } \\
\text { Sastourné }\end{array}$ & MingBooks & 2013 \\
\hline $\begin{array}{l}\text { Zhang Yihe 章诒和 } \\
(1942-)\end{array}$ & 杨氏女 & Madame Yang & $\begin{array}{l}\text { François } \\
\text { Sastourné }\end{array}$ & MingBooks & 2014 \\
\hline $\begin{array}{l}\text { Zhang Zezhong张泽忠 } \\
(1949-)\end{array}$ & 山乡笔记 & $\begin{array}{l}\text { Notes de régions } \\
\text { montagneuses }\end{array}$ & Annie Curien & Actes Sud & 2000 \\
\hline Zheng Yi 郑义 (1947-) & 肇论 & $\begin{array}{l}\text { Prière pour une âme } \\
\text { égarée }\end{array}$ & $\begin{array}{l}\text { Bernard } \\
\text { Bourrit, } \\
\text { Zhang Li }\end{array}$ & $\begin{array}{l}\text { Bleu de } \\
\text { Chine }\end{array}$ & 2007 \\
\hline $\begin{array}{l}\text { Zhu Wenying 朱文颖 } \\
\text { (1971-) }\end{array}$ & 无可替代的故事 & $\begin{array}{l}\text { L'incontournable } \\
\text { histoire, court roman }\end{array}$ & $\begin{array}{l}\text { Caroline } \\
\text { Grillot }\end{array}$ & $\begin{array}{l}\text { Bleu de } \\
\text { Chine }\end{array}$ & 2004 \\
\hline $\begin{array}{l}\text { Zhu Xining 朱天心 } \\
\text { (1958-) }\end{array}$ & 淡水最后列车 & $\begin{array}{l}\text { Le dernier train pour } \\
\text { Tamsui, et autres } \\
\text { nouvelles }\end{array}$ & $\begin{array}{l}\text { Angel Pino, } \\
\text { Isabelle Rabut }\end{array}$ & Bourgois & 2004 \\
\hline
\end{tabular}

